Acta Crystallographica Section B

Structural

Science

ISSN 0108-7681

\section{Hypothetical binodal zeolitic frameworks}

Alexandra Simperler, ${ }^{a}$ Martin D. Foster, ${ }^{a}$ Olaf Delgado Friedrichs, ${ }^{b}$ Robert G. Bell, ${ }^{a}$ Filipe A. Almeida $\operatorname{Paz}^{\mathrm{c}, \mathrm{d}}$ and Jacek Klinowski ${ }^{\mathrm{c}}$ *

aDavy-Faraday Research Laboratory, The Royal Institution of Great Britain, 21 Albemarle Street, London W1S 4BS, England, ${ }^{\mathbf{b}}$ Department of Chemistry and Biochemistry, Arizona State University, Tempe, AZ 85287-1604, USA, 'Department of Chemistry, University of Cambridge, Lensfield Road, Cambridge CB2 $1 \mathrm{EW}$, England, and dDepartment of Chemistry, CICECO, University of Aveiro, Campus Universitário de Santiago, Aveiro 3810-193, Portugal

Correspondence e-mail: jk18@cam.ac.uk
Hypothetical binodal zeolitic structures (structures containing two kinds of tetrahedral sites) were systematically enumerated using tiling theory and characterized by computational chemistry methods. Each of the 109 refineable topologies based on 'simple tilings' was converted into a silica polymorph and its energy minimized using the GULP program with the Sanders-Catlow silica potential. Optimized structural parameters, framework energies relative to $\alpha$-quartz and volumes accessible to sorption have been calculated. Eleven of the 30 known binodal topologies listed in the Atlas of Zeolite Framework Types were found, leaving 98 topologies that were unknown previously. The chemical feasibility of each structure as a zeolite was evaluated by means of a feasibility factor derived from the correlation between lattice energy and framework density. Structures are divided into 15 families, based on common structural features. Many 'feasible' structures contain only small pores. Several very open structures were also enumerated, although they contain three-membered rings which are thermodynamically disfavoured and not found in conventional zeolites. We believe that such topologies may be realizable as framework materials, but with different elemental compositions to those normally associated with zeolites.

\section{Introduction}

Zeolites find many important applications in science and technology in areas as diverse as catalysis, chemical separation, water softening, agriculture, refrigeration and optoelectronics. There are 152 distinct structural types of zeolites which have now been identified (Baerlocher et al., 2001). The definition of a zeolite is based not on chemical composition or function, but rather on atomic scale geometry. In order to qualify as a zeolite or zeolite-type material (zeotype), a mineral or synthetic material must possess a framework composed of corner-sharing tetrahedra. There is an additional requirement of 'openness', simultaneously dependent on density and smallest ring size, thus excluding denser minerals. Another way of expressing this is in terms of a four-connected net in which each vertex (in chemical terms the central atom of a tetrahedron) is connected to its four closest neighbours, normally via an oxygen bridge.

The enumeration of hypothetical zeolitic framework structures (Klinowski, 1998) is of considerable scientific and practical interest in terms of generating new nanoporous architectures. Enumeration originates with the work of Wells $(1977,1979,1984)$ on three-dimensional nets and polyhedra. Smith and collaborators (Smith, 1988, 1993; Alberti, 1979; Sato, 1984, 1987; Sherman \& Bennett, 1973; Barrer \& Villiger, 1969), O'Keeffe and collaborators (O'Keeffe \& Hyde,
Received 19 April 2005 Accepted 27 April 2005
(C) 2005 International Union of Crystallography Printed in Great Britain - all rights reserved 
1996a,b) and Akporiaye \& Price (1989) found many possible new structures by combining various structural subunits. More recent work involves computer search algorithms (Boisen et al., 1999; Treacy et al., 1997; Foster \& Treacy, 2004; MellotDraznieks et al., 2000).

Our work is based on advances in combinatorial tiling theory (Dress et al., 1993). A tiling is a periodic subdivision of three-dimensional space into connected regions, which we call tiles. If two tiles meet along a surface, the surface is called a face. If three or more faces meet along a curve, we call the curve an edge. If at least three edges meet at a point, we call that point a vertex. A network is thus formed by the vertices and edges. The configuration of edges, faces and tiles around a given vertex can be described via the so-called vertex figure, obtained by placing the centre of a small notational sphere at the vertex and considering the tiling of that sphere formed by the intersections with the different tiles touching that vertex. We have already enumerated all possible Euclidean uni-, biand trinodal tilings based on simple vertex figures and all uninodal tilings with vertex figures containing up to six extra edges (Delgado Friedrichs, 2001), and the computer program used for this task is available from the authors upon request (olaf.delgado@asu.edu).

The tiling approach identified networks with one, two and three types of inequivalent vertices, which we call uninodal, binodal and trinodal (Delgado Friedrichs et al., 1999). We have shown that there are exactly 9,117 and over 1300 topological types of four-connected uninodal, binodal and trinodal nets, respectively, which are based on 'simple' periodic tilings (as explained in Delgado Friedrichs et al., 1999). The previously reported number of 926 for the trinodal simple tilings included, due to an error in the manual processing of the data files, only those nets for which the tiles have non-trivial site symmetry. In addition, there are at least 157 additional uninodal nets derived from 'quasi-simple' tilings (the vertex figures of which are derived from tetrahedra, but contain double edges; Delgado Friedrichs et al., 1999) and which have already been discussed elsewhere (Foster et al., 2001, 2003; Foster, Friedrichs et al., 2004; Foster, Simperler et al., 2004; Simperler et al., 2004;). For example, zeolitic structure types SOD, LTA,
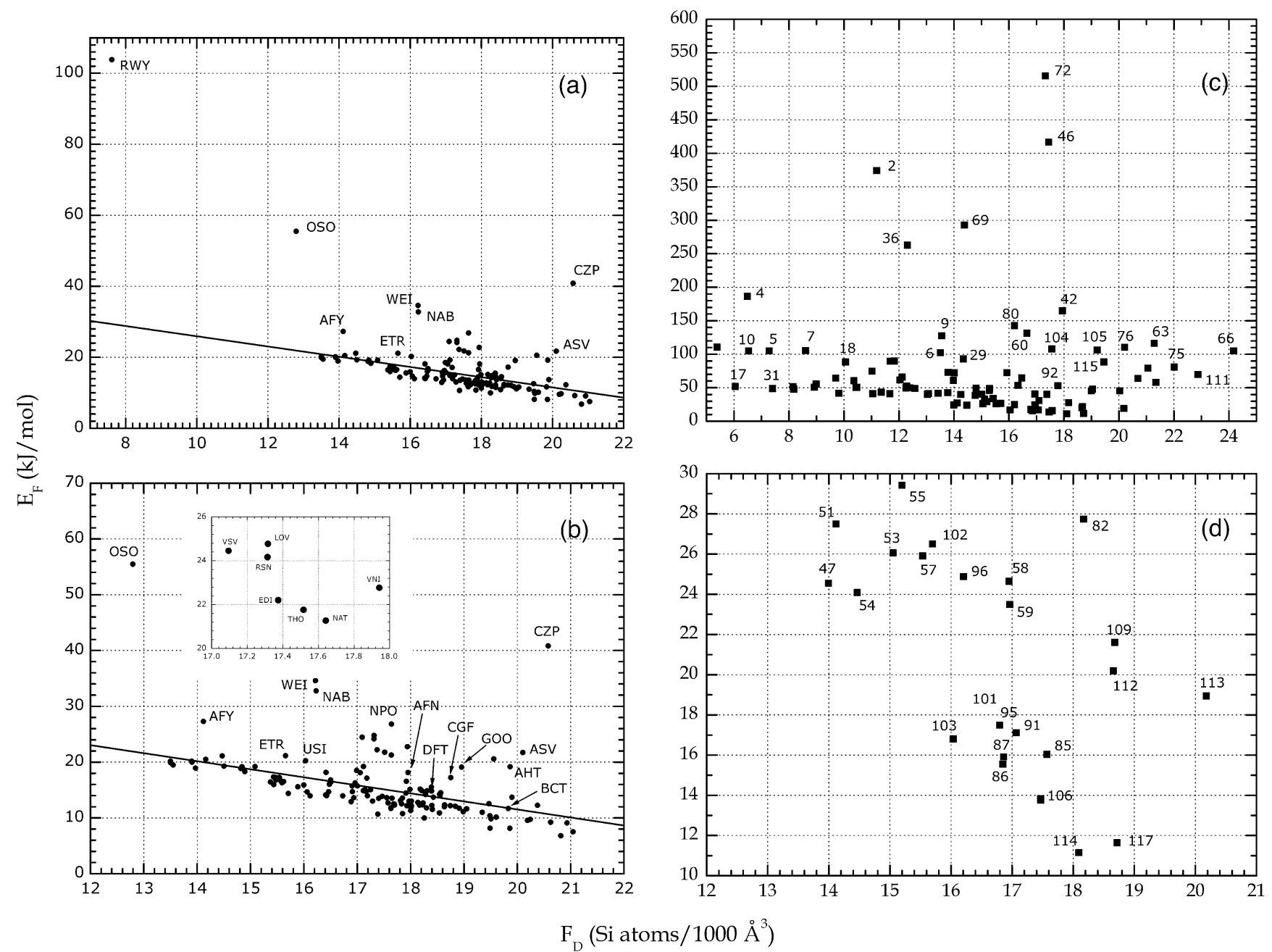

Figure 1

$\mathrm{F}_{\mathrm{D}}\left(\mathrm{Si}\right.$ atoms $\left./ 1000 \AA^{3}\right)$

Framework energy, $E_{F}\left(\mathrm{~kJ} \mathrm{~mol}^{-1}\right)$, with respect to $\alpha$-quartz, versus framework density, $F_{D}$ (Si atoms per $\left.1000 \AA^{3}\right)$, for $(a)$ and $(b)$ all known zeolitic structure types; $(c)$ and $(d)$ hypothetical binodal zeolitic structures. 
Table 1

Chemical feasibility factor, relative lattice energy, framework density and coordination sequences for 109 hypothetical binodal zeolites, optimized as purely siliceous structures.

Structures are listed in order of increasing value of $\vartheta$.

\begin{tabular}{|c|c|c|c|c|c|c|c|c|c|c|c|c|c|}
\hline \multirow{3}{*}{$\frac{\text { Structure }}{2 \_87}$} & \multirow{2}{*}{$\frac{\vartheta}{0.10}$} & \multirow{2}{*}{$\begin{array}{l}\begin{array}{l}\Delta E_{\text {quartz }} \\
\left(\mathrm{kJ} \mathrm{mol}^{-1}\right)\end{array} \\
15.91\end{array}$} & \multirow{2}{*}{$\begin{array}{l}F_{D} \\
(T \text { sites per } \\
\left.1000 \AA^{3}\right) \\
16.86\end{array}$} & \multicolumn{10}{|c|}{ Coordination sequence } \\
\hline & & & & 4 & 9 & 17 & 30 & 49 & 72 & 96 & 121 & 150 & 187 \\
\hline & & & & 4 & 10 & 20 & 33 & 49 & 69 & 94 & 125 & 160 & 197 \\
\hline \multirow[t]{2}{*}{ 2_89 (ERI) } & 0.12 & 16.39 & 16.51 & 4 & 9 & 17 & 30 & 50 & 75 & 98 & 118 & 144 & 185 \\
\hline & & & & 4 & 10 & 20 & 32 & 46 & 64 & 90 & 126 & 164 & 196 \\
\hline \multirow[t]{2}{*}{ 2_84 (EAB) } & 0.12 & 16.41 & 16.49 & 4 & 9 & 17 & 30 & 49 & 71 & 92 & 115 & 147 & 190 \\
\hline & & & & 4 & 10 & 20 & 32 & 46 & 66 & 94 & 128 & 162 & 192 \\
\hline \multirow[t]{2}{*}{ 2_90 (SAT) } & 0.18 & 15.72 & 16.91 & 4 & 9 & 17 & 30 & 50 & 75 & 100 & 126 & 157 & 194 \\
\hline & & & & 4 & 10 & 20 & 33 & 50 & 71 & 95 & 124 & 158 & 197 \\
\hline \multirow[t]{2}{*}{ 2_103 } & 0.30 & 16.80 & 16.04 & 4 & 10 & 17 & 30 & 52 & 70 & 107 & 128 & 166 & 208 \\
\hline & & & & 4 & 11 & 20 & 33 & 51 & 73 & 103 & 136 & 169 & 207 \\
\hline 2_88 (AWW) & 0.32 & 15.03 & 17.25 & 4 & 9 & 17 & 30 & 50 & 74 & 97 & 123 & 158 & 198 \\
\hline & & & & 4 & 10 & 20 & 33 & 50 & 72 & 98 & 128 & 162 & 200 \\
\hline $2 \_86$ & 0.37 & 15.54 & 16.85 & 4 & 9 & 17 & 30 & 49 & 72 & 96 & 121 & 150 & 186 \\
\hline & & & & 4 & 10 & 20 & 33 & 49 & 68 & 92 & 122 & 155 & 191 \\
\hline 2_83 (LEV) & 0.42 & 16.00 & 16.48 & 4 & 9 & 17 & 30 & 49 & 71 & 92 & 114 & 143 & 183 \\
\hline & & & & 4 & 10 & 20 & 32 & 46 & 64 & 90 & 124 & 156 & 184 \\
\hline $2 \_85$ & 0.69 & 16.03 & 17.57 & 4 & 9 & 17 & 30 & 49 & 71 & 95 & 125 & 161 & 201 \\
\hline & & & & 4 & 10 & 20 & 33 & 50 & 73 & 100 & 131 & 168 & 208 \\
\hline 2_107 (LOS) & 0.91 & 13.86 & 17.47 & 4 & 10 & 20 & 34 & 52 & 74 & 102 & 136 & 172 & 210 \\
\hline & & & & 4 & 10 & 20 & 34 & 54 & 78 & 104 & 134 & 168 & 210 \\
\hline 2_74 (TSC) & 0.94 & 19.47 & 13.55 & 4 & 9 & 16 & 25 & 37 & 53 & 74 & 99 & 125 & 151 \\
\hline & & & & 4 & 9 & 17 & 28 & 41 & 56 & 73 & 93 & 117 & 146 \\
\hline 2_110 & 0.94 & 13.82 & 17.47 & 4 & 9 & 17 & 30 & 50 & 74 & 97 & 123 & 158 & 198 \\
\hline & & & & 4 & 10 & 20 & 33 & 50 & 72 & 98 & 128 & 162 & 200 \\
\hline 2_106 & 0.97 & 13.79 & 17.46 & 4 & 10 & 20 & 34 & 52 & 74 & 100 & 130 & 166 & 208 \\
\hline & & & & 4 & 10 & 20 & 34 & 53 & 76 & 103 & 135 & 170 & 209 \\
\hline 2_95 & 0.93 & 17.49 & 16.80 & 4 & 9 & 18 & 32 & 52 & 75 & 99 & 133 & 171 & 207 \\
\hline & & & & 4 & 10 & 19 & 32 & 52 & 76 & 103 & 136 & 172 & 213 \\
\hline 2_108 & 0.97 & 13.77 & 17.47 & 4 & 10 & 20 & 34 & 53 & 76 & 102 & 132 & 167 & 208 \\
\hline & & & & 4 & 10 & 20 & 34 & 53 & 76 & 103 & 135 & 170 & 208 \\
\hline 2_81 (SAS) & 0.98 & 15.88 & 16.00 & 4 & 9 & 17 & 30 & 48 & 68 & 87 & 109 & 142 & 184 \\
\hline & & & & 4 & 10 & 19 & 30 & 45 & 65 & 90 & 118 & 145 & 175 \\
\hline 2_91 & 0.95 & 17.12 & 17.07 & 4 & 9 & 17 & 31 & 54 & 82 & 108 & 137 & 176 & 223 \\
\hline & & & & 4 & 11 & 22 & 35 & 55 & 81 & 107 & 143 & 184 & 222 \\
\hline 2_78 (AFX) & 1.00 & 16.41 & 15.61 & 4 & 9 & 17 & 29 & 45 & 64 & 85 & 110 & 141 & 178 \\
\hline & & & & 4 & 9 & 17 & 29 & 45 & 65 & 89 & 116 & 144 & 175 \\
\hline 2_101 (AST) & 0.99 & 18.14 & 16.41 & 4 & 9 & 19 & 34 & 48 & 66 & 96 & 127 & 151 & 183 \\
\hline & & & & 4 & 12 & 18 & 28 & 52 & 78 & 88 & 112 & 162 & 204 \\
\hline 2_117 & 1.22 & 11.58 & 18.74 & 4 & 11 & 24 & 41 & 64 & 93 & 127 & 163 & 205 & 255 \\
\hline & & & & 4 & 12 & 22 & 44 & 64 & 94 & 124 & 164 & 206 & 252 \\
\hline 2_114 & 2.17 & 11.15 & 18.09 & 4 & 11 & 21 & 36 & 64 & 93 & 120 & 156 & 202 & 255 \\
\hline & & & & 4 & 11 & 23 & 40 & 62 & 88 & 123 & 162 & 202 & 249 \\
\hline 2_47 & 3.02 & 24.55 & 14.00 & 4 & 9 & 17 & 28 & 41 & 56 & 74 & 97 & 125 & 158 \\
\hline & & & & 4 & 8 & 14 & 24 & 37 & 54 & 75 & 97 & 121 & 148 \\
\hline $2 \_54$ & 3.18 & 24.09 & 14.47 & 4 & 8 & 14 & 25 & 40 & 57 & 76 & 96 & 119 & 150 \\
\hline & & & & 4 & 9 & 17 & 27 & 38 & 54 & 76 & 101 & 128 & 154 \\
\hline 2_112 & 4.66 & 20.19 & 18.66 & 4 & 10 & 22 & 40 & 60 & 95 & 121 & 165 & 212 & 258 \\
\hline & & & & 4 & 12 & 21 & 41 & 67 & 90 & 128 & 168 & 211 & 263 \\
\hline 2_50 (AFY) & 5.03 & 27.27 & 14.12 & 4 & 8 & 14 & 25 & 39 & 53 & 71 & 96 & 124 & 152 \\
\hline & & & & 4 & 9 & 16 & 23 & 34 & 57 & 82 & 98 & 115 & 141 \\
\hline $2 \_53$ & 5.11 & 26.05 & 15.05 & 4 & 7 & 12 & 24 & 39 & 60 & 79 & 110 & 168 & 250 \\
\hline & & & & 4 & 10 & 19 & 27 & 39 & 62 & 92 & 137 & 202 & 275 \\
\hline 2_51 & 5.18 & 27.49 & 14.12 & 4 & 8 & 14 & 25 & 39 & 53 & 72 & 100 & 130 & 157 \\
\hline & & & & 4 & 9 & 16 & 23 & 34 & 57 & 82 & 98 & 118 & 153 \\
\hline 2_59 & 5.25 & 23.49 & 16.96 & 4 & 9 & 18 & 32 & 52 & 75 & 99 & 133 & 171 & 207 \\
\hline & & & & 4 & 10 & 19 & 32 & 52 & 76 & 103 & 136 & 172 & 213 \\
\hline 2_113 & 5.32 & 18.94 & 20.19 & 4 & 10 & 23 & 38 & 60 & 86 & 118 & 154 & 195 & 244 \\
\hline & & & & 4 & 11 & 21 & 39 & 61 & 86 & 118 & 154 & 195 & 243 \\
\hline 2_96 & 5.45 & 24.88 & 16.21 & 4 & 9 & 18 & 32 & 52 & 75 & 105 & 144 & 181 & 217 \\
\hline & & & & 4 & 11 & 21 & 35 & 54 & 80 & 113 & 145 & 182 & 228 \\
\hline 2_57 & 5.51 & 25.91 & 15.54 & 4 & 8 & 14 & 26 & 44 & 63 & 80 & 97 & 122 & 164 \\
\hline & & & & 4 & 10 & 19 & 28 & 39 & 57 & 82 & 112 & 139 & 159 \\
\hline 2_109 & 5.67 & 21.61 & 18.68 & 4 & 10 & 20 & 34 & 53 & 76 & 102 & 133 & 170 & 212 \\
\hline & & & & 4 & 10 & 20 & 34 & 53 & 77 & 106 & 139 & 174 & 212 \\
\hline 2_58 & 6.04 & 24.64 & 16.95 & 4 & 8 & 14 & 26 & 45 & 67 & 89 & 115 & 149 & 188 \\
\hline & & & & 4 & 10 & 20 & 32 & 47 & 68 & 93 & 122 & 157 & 196 \\
\hline
\end{tabular}

RHO, FAU, KFI and CHA are all based on quasi-simple tilings. An example of a non-simple tiling is that of GIS, where the tile has some two-connected vertices.

Here we focus our attention on the binodal structures, i.e. those with two topologically inequivalent types of tetrahedral vertex ( $T$-atom sites) derived only from simple tilings, meaning that they can be readily described by the packing of convex polyhedra, the vertices of which are all three-connected. Structures containing cages are thus found in abundance, while those with, for instance, more 'cylindrical' channels are less common and tend to have lower framework density than the 'quasisimple' structures, with a greater proportion lying in the range of density where most known zeolites are found, as opposed to denser minerals. On the other hand, many of the known zeolite structure types cannot be constructed from simple tilings. Thus, simple tilings cannot generate the complete set of binodal zeolites. Seven of the 21 known uninodal zeolites correspond to simple tilings, and the remaining 14 , together with several mineral structures (although not quartz) are constructed using quasi-simple tilings. We have found 11 of the 30 known binodal zeolite types, and the remaining 19 will be found by considering quasi-simple tilings, just as with the uninodal structures. The number of potential binodal networks thus generated will be enormous, and their enumeration will require the use of state-of-the-art computational facilities. However, only very few binodal structures have previously been enumerated, while nearly all uninodal structures derived from the tilings were previously known, either as crystal structures or as hypothetical nets. It is therefore of interest to describe the binodal structures derived only from simple tilings.

To characterize the structures, we follow procedures identical to 
Table 1 (continued)

\begin{tabular}{|c|c|c|c|c|c|c|c|c|c|c|c|c|c|}
\hline \multirow{2}{*}{$\frac{\text { Structure }}{2 \_102}$} & \multirow{2}{*}{$\frac{\vartheta}{6.08}$} & \multirow{2}{*}{$\begin{array}{l}\begin{array}{l}\Delta E_{\text {quartz }} \\
\left(\mathrm{kJ} \mathrm{mol}^{-1}\right)\end{array} \\
26.50\end{array}$} & \multirow{2}{*}{$\begin{array}{l}F_{D} \\
(T \text { sites per } \\
\left.1000 \AA^{3}\right) \\
15.70\end{array}$} & \multicolumn{5}{|c|}{ Coordination sequence } & \multirow[b]{2}{*}{73} & \multirow[b]{2}{*}{98} & & & \\
\hline & & & & 4 & 9 & 19 & 34 & 48 & & & 125 & 167 & 197 \\
\hline & & & & 4 & 11 & 18 & 31 & 54 & 72 & 96 & 128 & 160 & 204 \\
\hline $2 \_55$ & 7.61 & 29.43 & 15.20 & 4 & 8 & 14 & 26 & 44 & 62 & 91 & 121 & 144 & 181 \\
\hline & & & & 4 & 11 & 19 & 29 & 47 & 67 & 91 & 121 & 153 & 188 \\
\hline $2 \_82$ & 9.41 & 27.74 & 18.17 & 4 & 9 & 17 & 30 & 48 & 69 & 92 & 119 & 153 & 192 \\
\hline & & & & 4 & 10 & 20 & 32 & 46 & 66 & 94 & 126 & 158 & 194 \\
\hline 2_67 & 10.27 & 33.41 & 15.11 & 4 & 8 & 16 & 28 & 42 & 60 & 84 & 108 & 136 & 170 \\
\hline & & & & 4 & 9 & 16 & 27 & 43 & 62 & 83 & 109 & 139 & 171 \\
\hline 2_99 & 10.53 & 30.91 & 17.10 & 4 & 9 & 18 & 34 & 55 & 76 & 103 & 144 & 187 & 229 \\
\hline & & & & 4 & 9 & 20 & 34 & 54 & 81 & 110 & 144 & 185 & 229 \\
\hline 2_35 & 10.69 & 41.65 & 9.82 & 4 & 8 & 13 & 20 & 28 & 36 & 46 & 62 & 83 & 104 \\
\hline & & & & 4 & 9 & 15 & 21 & 28 & 37 & 49 & 65 & 85 & 108 \\
\hline 2_62 & 10.97 & 33.96 & 15.43 & 4 & 8 & 14 & 27 & 48 & 70 & 91 & 116 & 146 & 185 \\
\hline & & & & 4 & 9 & 19 & 32 & 45 & 67 & 92 & 124 & 165 & 209 \\
\hline 2_43 & 11.62 & 41.22 & 11.05 & 4 & 8 & 14 & 21 & 34 & 53 & 71 & 90 & 108 & 133 \\
\hline & & & & 4 & 8 & 16 & 27 & 35 & 48 & 66 & 83 & 113 & 146 \\
\hline 2_64 & 12.15 & 41.09 & 11.67 & 4 & 8 & 15 & 25 & 37 & 52 & 71 & 95 & 120 & 148 \\
\hline & & & & 4 & 8 & 16 & 27 & 37 & 53 & 71 & 89 & 116 & 144 \\
\hline 2_45 & 12.91 & 40.22 & 13.03 & 4 & 8 & 14 & 23 & 34 & 49 & 67 & 87 & 111 & 139 \\
\hline & & & & 4 & 9 & 16 & 25 & 37 & 52 & 70 & 91 & 114 & 140 \\
\hline 2_31 & 13.16 & 48.71 & 7.40 & 4 & 8 & 12 & 17 & 24 & 31 & 36 & 42 & 54 & 72 \\
\hline & & & & 4 & 9 & 15 & 20 & 24 & 29 & 37 & 48 & 60 & 73 \\
\hline 2_24 & 13.28 & 43.16 & 11.36 & 4 & 7 & 12 & 22 & 32 & 41 & 56 & 80 & 106 & 125 \\
\hline & & & & 4 & 9 & 15 & 22 & 32 & 46 & 63 & 81 & 100 & 122 \\
\hline 2_19 & 13.39 & 47.93 & 8.17 & 4 & 7 & 10 & 16 & 22 & 26 & 34 & 48 & 63 & 76 \\
\hline & & & & 4 & 8 & 12 & 16 & 21 & 28 & 37 & 49 & 64 & 80 \\
\hline 2_73 & 13.62 & 38.71 & 14.78 & 4 & 9 & 15 & 21 & 37 & 59 & 104 & 138 & 182 & 199 \\
\hline & & & & 4 & 11 & 20 & 36 & 52 & 77 & 121 & 155 & 192 & 236 \\
\hline 2_68 & 13.82 & 41.46 & 13.08 & 4 & 8 & 17 & 28 & 45 & 66 & 88 & 114 & 141 & 182 \\
\hline & & & & 4 & 9 & 16 & 28 & 48 & 66 & 84 & 115 & 150 & 178 \\
\hline 2_39 & 13.86 & 39.83 & 14.25 & 4 & 8 & 13 & 22 & 36 & 53 & 72 & 94 & 122 & 156 \\
\hline & & & & 4 & 9 & 16 & 25 & 38 & 56 & 78 & 103 & 129 & 157 \\
\hline 2_17 & 13.96 & 51.80 & 6.05 & 4 & 7 & 9 & 13 & 19 & 23 & 25 & 30 & 41 & 55 \\
\hline & & & & 4 & 8 & 12 & 15 & 17 & 21 & 28 & 36 & 44 & 53 \\
\hline 2_70 & 14.33 & 41.70 & 13.42 & 4 & 8 & 17 & 32 & 46 & 71 & 95 & 129 & 166 & 199 \\
\hline & & & & 4 & 9 & 18 & 32 & 50 & 70 & 95 & 128 & 166 & 212 \\
\hline 2_40 & 14.97 & 40.32 & 15.02 & 4 & 8 & 13 & 22 & 37 & 56 & 76 & 98 & 126 & 158 \\
\hline & & & & 4 & 9 & 16 & 26 & 41 & 60 & 80 & 101 & 126 & 158 \\
\hline 2_27 & 15.42 & 42.76 & 13.78 & 4 & 7 & 12 & 24 & 38 & 50 & 68 & 94 & 122 & 153 \\
\hline & & & & 4 & 9 & 16 & 26 & 40 & 57 & 78 & 103 & 130 & 159 \\
\hline 2_23 & 15.97 & 51.69 & 8.14 & 4 & 7 & 12 & 20 & 26 & 32 & 44 & 68 & 90 & 108 \\
\hline & & & & 4 & 8 & 13 & 17 & 24 & 34 & 49 & 67 & 82 & 101 \\
\hline 2_20 & 16.11 & 50.76 & 8.93 & 4 & 7 & 10 & 17 & 27 & 35 & 41 & 52 & 73 & 100 \\
\hline & & & & 4 & 9 & 15 & 20 & 25 & 33 & 47 & 66 & 84 & 98 \\
\hline 2_97 & 16.99 & 40.46 & 16.94 & 4 & 9 & 18 & 32 & 52 & 76 & 106 & 147 & 188 & 229 \\
\hline & & & & 4 & 11 & 21 & 35 & 55 & 81 & 117 & 152 & 188 & 238 \\
\hline 2_71 & 17.07 & 39.95 & 17.38 & 4 & 8 & 19 & 39 & 58 & 83 & 118 & 160 & 193 & 232 \\
\hline & & & & 4 & 10 & 21 & 38 & 58 & 91 & 117 & 158 & 195 & 244 \\
\hline 2_26 & 17.51 & 50.60 & 10.44 & 4 & 7 & 12 & 22 & 34 & 46 & 58 & 76 & 107 & 139 \\
\hline & & & & 4 & 8 & 14 & 21 & 32 & 48 & 65 & 86 & 111 & 138 \\
\hline 2_25 & 17.56 & 50.63 & 10.47 & 4 & 7 & 12 & 22 & 33 & 44 & 58 & 80 & 104 & 125 \\
\hline & & & & 4 & 8 & 14 & 21 & 32 & 48 & 65 & 85 & 106 & 132 \\
\hline 2_37 & 18.50 & 48.93 & 12.58 & 4 & 8 & 12 & 17 & 24 & 31 & 36 & 42 & 54 & 72 \\
\hline & & & & 4 & 9 & 15 & 20 & 24 & 29 & 37 & 48 & 60 & 73 \\
\hline 2_21 & 18.85 & 49.90 & 12.26 & 4 & 7 & 10 & 18 & 32 & 47 & 59 & 71 & 91 & 121 \\
\hline & & & & 4 & 9 & 16 & 24 & 34 & 48 & 66 & 89 & 117 & 149 \\
\hline 2_32 & 19.04 & 49.98 & 12.40 & 4 & 8 & 12 & 18 & 29 & 44 & 60 & 77 & 98 & 125 \\
\hline & & & & 4 & 9 & 16 & 24 & 33 & 45 & 62 & 85 & 113 & 143 \\
\hline 2_48 & 19.09 & 45.87 & 15.29 & 4 & 8 & 14 & 25 & 38 & 50 & 70 & 100 & 125 & 147 \\
\hline & & & & 4 & 9 & 16 & 24 & 36 & 56 & 76 & 92 & 120 & 159 \\
\hline 2_41 & 19.44 & 55.48 & 8.99 & 4 & 8 & 14 & 19 & 26 & 40 & 52 & 70 & 88 & 100 \\
\hline & & & & 4 & 8 & 14 & 20 & 29 & 42 & 52 & 68 & 89 & 109 \\
\hline 2_69 & 20.57 & 49.50 & 14.25 & 4 & 8 & 17 & 29 & 46 & 68 & 91 & 117 & 154 & 184 \\
\hline & & & & 4 & 9 & 17 & 28 & 49 & 69 & 92 & 119 & 151 & 184 \\
\hline 2_65 & 20.64 & 52.45 & 12.28 & 4 & 8 & 15 & 28 & 47 & 66 & 86 & 118 & 155 & 181 \\
\hline & & & & 4 & 8 & 16 & 26 & 48 & 66 & 88 & 120 & 142 & 200 \\
\hline 2_33 & 20.83 & 49.10 & 14.80 & 4 & 8 & 12 & 18 & 30 & 49 & 71 & 92 & 114 & 143 \\
\hline & & & & 4 & 9 & 16 & 25 & 38 & 56 & 77 & 99 & 121 & 147 \\
\hline $2 \_52$ & 20.93 & 48.51 & 15.30 & 4 & 7 & 10 & 16 & 25 & 34 & 43 & 58 & 75 & 90 \\
\hline & & & & 4 & 7 & 11 & 16 & 24 & 35 & 46 & 59 & 75 & 93 \\
\hline
\end{tabular}

those used in our previous work (Foster et al., 2003; Foster, Simperler et al., 2004). These involve generating model $\mathrm{SiO}_{2}$ polymorphs from the tiling nets and optimizing them using lattice energy minimization. Apart from obtaining an optimized structure for each topology, we also calculate a lattice energy, which provides an accurate guide to the thermodynamic stability that such a phase might have. A 'feasibility factor', $\vartheta$, derived from the correlation between lattice energy and density calculated for known zeolite structure types, serves as a further measure of thermodynamic feasibility. We have also calculated the accessible volume for each pore system using a standard definition (Molecular Simulations Inc., 1999).

In describing the structural characteristics of each framework, we have resorted to the 'model building' approach (Baerlocher et al., 2001; Smith, 1988; Meier, 1986; Liebau et al., 1986), which is consistent with descriptions found in the online zeolite database and allows structures to be classified into 'families' if they share certain structural motifs. As part of this analysis we define as a composite building unit (CBU) every small finite unit from which a structure may be generated. These units can be corner-, edge- or face-sharing, or joined to one another by single linkages. The automated assembly of such units is also a potential method of structural enumeration, as demonstrated by Mellot-Draznieks (Mellot-Draznieks et al., 2000, 2002). Zeolite structures may also be described in terms of the strictly defined secondary building units (SBUs), one type of which may be used to build a unit cell of the zeolite, without sharing $T$ atoms. Here, we have not used the SBU approach, finding it more informative to use alternative descriptions (in general, our building units tend to be larger). However, the SBUs involved may be readily identified, as may the 
Table 1 (continued)

\begin{tabular}{|c|c|c|c|c|c|c|c|c|c|c|c|c|c|}
\hline Structure & $\vartheta$ & $\begin{array}{l}\Delta E_{\text {quartz }} \\
\left(\mathrm{kJ} \mathrm{mol}^{-1}\right)\end{array}$ & $\begin{array}{l}F_{D} \\
(T \text { sites per } \\
\left.1000 \AA^{3}\right)\end{array}$ & & ordin & tion & sequ & nce & & & & & \\
\hline \multirow[t]{2}{*}{ 2_100 } & 22.42 & 45.32 & 19.00 & 4 & 9 & 18 & 34 & 58 & 86 & 113 & 146 & 194 & 248 \\
\hline & & & & 4 & 11 & 22 & 38 & 61 & 88 & 120 & 157 & 199 & 246 \\
\hline \multirow[t]{2}{*}{ 2_79 } & 23.48 & 45.37 & 20.03 & 4 & 9 & 17 & 29 & 46 & 69 & 98 & 133 & 174 & 221 \\
\hline & & & & 4 & 10 & 21 & 37 & 58 & 84 & 114 & 148 & 186 & 229 \\
\hline \multirow[t]{2}{*}{$2 \_77$} & 24.27 & 47.94 & 19.04 & 4 & 9 & 16 & 26 & 41 & 61 & 84 & 110 & 140 & 175 \\
\hline & & & & 4 & 9 & 17 & 28 & 42 & 61 & 85 & 114 & 146 & 179 \\
\hline \multirow[t]{2}{*}{$2 \_44$} & 24.31 & 60.53 & 10.36 & 4 & 8 & 14 & 21 & 36 & 55 & 75 & 94 & 120 & 154 \\
\hline & & & & 4 & 8 & 16 & 20 & 34 & 64 & 72 & 96 & 128 & 146 \\
\hline \multirow[t]{2}{*}{ 2_46 } & 24.45 & 57.04 & 12.91 & 4 & 8 & 14 & 24 & 36 & 48 & 64 & 90 & 118 & 136 \\
\hline & & & & 4 & 9 & 15 & 22 & 34 & 52 & 71 & 87 & 106 & 136 \\
\hline \multirow[t]{2}{*}{ 2_61 } & 25.45 & 53.55 & 16.33 & 4 & 8 & 14 & 26 & 46 & 70 & 91 & 113 & 149 & 197 \\
\hline & & & & 4 & 10 & 19 & 30 & 45 & 68 & 94 & 122 & 152 & 186 \\
\hline \multirow[t]{2}{*}{ 2_22 } & 26.31 & 64.37 & 9.70 & 4 & 7 & 11 & 18 & 28 & 42 & 56 & 68 & 85 & 111 \\
\hline & & & & 4 & 8 & 14 & 21 & 29 & 41 & 57 & 77 & 99 & 121 \\
\hline 2_92 & 26.57 & 53.08 & 17.78 & 4 & 9 & 17 & 31 & 54 & 82 & 109 & 139 & 182 & 233 \\
\hline & & & & 4 & 11 & 22 & 35 & 55 & 82 & 110 & 146 & 188 & 230 \\
\hline 2_13 & 26.62 & 61.44 & 12.04 & 4 & 6 & 15 & 28 & 34 & 60 & 69 & 96 & 126 & 142 \\
\hline & & & & 4 & 9 & 16 & 25 & 39 & 57 & 75 & 96 & 120 & 150 \\
\hline 2_93 & 28.25 & 60.99 & 13.98 & 4 & 10 & 20 & 31 & 50 & 71 & 104 & 134 & 176 & 210 \\
\hline & & & & 4 & 9 & 18 & 30 & 48 & 70 & 94 & 134 & 180 & 213 \\
\hline 2_12 & 29.67 & 65.74 & 12.11 & 4 & 6 & 15 & 20 & 30 & 50 & 67 & 90 & 115 & 126 \\
\hline & & & & 4 & 8 & 13 & 22 & 32 & 47 & 71 & 91 & 108 & 132 \\
\hline 2_30 & 33.21 & 64.55 & 16.47 & 4 & 8 & 12 & 16 & 26 & 42 & 56 & 72 & 102 & 140 \\
\hline & & & & 4 & 8 & 13 & 20 & 30 & 41 & 56 & 80 & 111 & 138 \\
\hline 2_94 & 33.62 & 58.11 & 21.34 & 4 & 9 & 18 & 31 & 55 & 88 & 121 & 157 & 194 & 236 \\
\hline & & & & 4 & 11 & 23 & 41 & 63 & 88 & 123 & 162 & 207 & 262 \\
\hline 2_16 & 36.19 & 72.38 & 14.02 & 4 & 6 & 17 & 32 & 49 & 65 & 92 & 135 & 167 & 183 \\
\hline & & & & 4 & 11 & 20 & 28 & 50 & 81 & 102 & 117 & 159 & 222 \\
\hline 2_34 & 36.28 & 76.83 & 11.03 & 4 & 8 & 13 & 19 & 26 & 38 & 55 & 74 & 95 & 115 \\
\hline & & & & 4 & 9 & 16 & 24 & 34 & 47 & 61 & 78 & 100 & 126 \\
\hline 2_14 & 36.36 & 72.96 & 13.79 & 4 & 6 & 16 & 31 & 48 & 57 & 77 & 116 & 154 & 161 \\
\hline & & & & 4 & 11 & 19 & 26 & 42 & 70 & 93 & 103 & 128 & 182 \\
\hline 2_98 & 37.01 & 63.95 & 20.69 & 4 & 9 & 18 & 33 & 51 & 72 & 105 & 147 & 184 & 230 \\
\hline & & & & 4 & 9 & 18 & 33 & 53 & 78 & 108 & 143 & 184 & 232 \\
\hline 2_56 & 38.29 & 72.66 & 15.93 & 4 & 8 & 14 & 26 & 44 & 62 & 93 & 122 & 145 & 182 \\
\hline & & & & 4 & 11 & 19 & 29 & 47 & 68 & 94 & 123 & 155 & 193 \\
\hline 2_116 & 43.29 & 71.75 & 21.56 & 4 & 11 & 22 & 39 & 65 & 96 & 134 & 175 & 223 & 280 \\
\hline & & & & 4 & 11 & 23 & 41 & 65 & 94 & 133 & 177 & 230 & 284 \\
\hline 2_111 & 43.36 & 69.96 & 22.87 & 4 & 10 & 20 & 46 & 70 & 94 & 140 & 206 & 264 & 308 \\
\hline & & & & 4 & 12 & 25 & 47 & 74 & 108 & 155 & 203 & 262 & 334 \\
\hline 2_18 & 44.29 & 89.79 & 10.06 & 4 & 7 & 10 & 14 & 17 & 24 & 37 & 48 & 57 & 70 \\
\hline & & & & 4 & 8 & 8 & 10 & 20 & 24 & 28 & 50 & 64 & 64 \\
\hline 2_28 & 45.34 & 88.96 & 11.69 & 4 & 7 & 13 & 18 & 33 & 44 & 66 & 72 & 110 & 118 \\
\hline & & & & 4 & 8 & 12 & 21 & 30 & 50 & 58 & 82 & 98 & 138 \\
\hline 2_15 & 46.13 & 89.89 & 11.83 & 4 & 12 & 10 & 28 & 52 & 34 & 84 & 124 & 74 & 172 \\
\hline & & & & 4 & 6 & 17 & 27 & 31 & 64 & 75 & 81 & 143 & 146 \\
\hline 2_75 & 50.08 & 80.91 & 22.01 & 4 & 7 & 13 & 25 & 39 & 56 & 87 & 107 & 148 & 182 \\
\hline & & & & 4 & 8 & 14 & 25 & 40 & 59 & 84 & 110 & 147 & 180 \\
\hline 2_29 & 50.83 & 93.06 & 14.34 & 4 & 10 & 18 & 30 & 45 & 59 & 103 & 165 & 219 & 314 \\
\hline & & & & 4 & 10 & 20 & 31 & 49 & 80 & 103 & 164 & 269 & 289 \\
\hline 2_10 & 51.21 & 104.88 & 6.53 & 4 & 6 & 12 & 16 & 24 & 32 & 44 & 55 & 68 & 80 \\
\hline & & & & 4 & 6 & 12 & 17 & 24 & 31 & 44 & 55 & 68 & 82 \\
\hline $2 \_5$ & 52.01 & 104.95 & 7.28 & 4 & 5 & 9 & 14 & 13 & 16 & 26 & 34 & 36 & 44 \\
\hline & & & & 4 & 8 & 10 & 11 & 16 & 22 & 24 & 28 & 42 & 60 \\
\hline 2_115 & 52.70 & 88.37 & 19.46 & 4 & 11 & 21 & 36 & 64 & 94 & 123 & 165 & 214 & 272 \\
\hline & & & & 4 & 11 & 23 & 40 & 63 & 91 & 126 & 167 & 213 & 265 \\
\hline $2 \_7$ & 53.66 & 105.42 & 8.60 & 4 & 5 & 10 & 20 & 26 & 24 & 44 & 80 & 98 & 93 \\
\hline & & & & 4 & 9 & 14 & 16 & 22 & 40 & 58 & 72 & 83 & 109 \\
\hline $2 \_8$ & 54.06 & 110.45 & 5.39 & 4 & 6 & 7 & 12 & 19 & 21 & 22 & 30 & 46 & 58 \\
\hline & & & & 4 & 8 & 12 & 13 & 16 & 22 & 30 & 36 & 44 & 56 \\
\hline 2_6 & 56.38 & 102.26 & 13.51 & 4 & 5 & 10 & 19 & 22 & 25 & 40 & 62 & 80 & 90 \\
\hline & & & & 4 & 9 & 13 & 16 & 23 & 36 & 50 & 58 & 68 & 94 \\
\hline 2_104 & 64.22 & 107.73 & 17.56 & 4 & 10 & 17 & 30 & 52 & 72 & 108 & 130 & 167 & 208 \\
\hline & & & & 4 & 11 & 20 & 33 & 52 & 76 & 105 & 138 & 173 & 213 \\
\hline 2_105 & 65.06 & 106.55 & 19.22 & 4 & 6 & 9 & 15 & 28 & 43 & 65 & 92 & 134 & 172 \\
\hline & & & & 4 & 7 & 11 & 20 & 31 & 47 & 74 & 99 & 133 & 196 \\
\hline $2 \_76$ & 68.64 & 110.29 & 20.21 & 4 & 9 & 16 & 25 & 38 & 58 & 87 & 124 & 165 & 209 \\
\hline & & & & 4 & 10 & 20 & 34 & 53 & 78 & 109 & 146 & 191 & 245 \\
\hline $2 \_63$ & 73.83 & 116.23 & 21.28 & 4 & 8 & 14 & 27 & 50 & 80 & 114 & 153 & 200 & 258 \\
\hline & & & & 4 & 11 & 23 & 39 & 62 & 93 & 130 & 174 & 223 & 275 \\
\hline
\end{tabular}

infinite periodic building units (PerBUs). We note that none of the units discussed are intended to represent the precursors from which zeolite crystals grow; neither do they necessarily correspond to the tiles of the original nets.

We discuss the structures in terms of the component units, and relate these to the calculated stability and feasibility. Taken together, thermodynamic feasibilty and the nature of the building units can provide a good initial guide as to which of these structures could be most readily synthesized.

\section{Energy minimization}

The systematically enumerated nets (Delgado Friedrichs et al., 1999) were first converted into atomistic models. This was done by inserting an $\mathrm{Si}$ atom at each vertex point in the network and placing a bridging oxygen between each pair of adjacent Si atoms. Each net was scaled such that the vertices were separated by ca $3.1 \AA$, a typical $\mathrm{Si}-$ Si distance. The resulting structure was then pre-optimized using the DLS (distance least squares) method (Meier \& Villiger, 1969), which performs geometric refinement of the structure by fitting bond lengths and angles to the prescribed values, and reduces the amount of computer time needed for the subsequent minimization of lattice energy. This procedure was found to have no influence on the final result: using lattice energy minimization from the outset gives the same structure, but at greater computational expense. The lattice energy and crystallographic data are those extracted from the GULP minimizations, whereas coordination sequences, bond distances and angles were calculated with zeoTsites (Version 1.2; Sastre \& Gale, 2001). The connectivity was additionally checked with the software tool KRIBER (Version 1.1; Bialek, 1995). Additional calculations were carried out using Cerius $^{2}$ software (Molecular Simulations Inc., 1999). 
Table 1 (continued)

\begin{tabular}{|c|c|c|c|c|c|c|c|c|c|c|c|c|c|}
\hline \multirow{2}{*}{$\frac{\text { Structure }}{2 \_9}$} & \multirow{2}{*}{$\frac{\vartheta}{73.85}$} & \multirow{2}{*}{$\begin{array}{l}\begin{array}{l}\Delta E_{\text {quartz }} \\
\left(\mathrm{kJ} \mathrm{mol}^{-1}\right)\end{array} \\
127.41\end{array}$} & \multirow{2}{*}{$\begin{array}{l}F_{D} \\
(T \text { sites per } \\
\left.1000 \AA^{3}\right) \\
13.56\end{array}$} & \multicolumn{10}{|c|}{ Coordination sequence } \\
\hline & & & & 4 & 6 & 8 & 14 & 20 & 30 & 45 & 54 & 73 & 98 \\
\hline & & & & 4 & 7 & 10 & 14 & 22 & 34 & 42 & 58 & 78 & 94 \\
\hline \multirow[t]{2}{*}{ 2_60 } & 79.89 & 131.67 & 16.65 & 4 & 8 & 14 & 26 & 45 & 68 & 93 & 125 & 171 & 223 \\
\hline & & & & 4 & 11 & 22 & 35 & 52 & 76 & 109 & 148 & 189 & 232 \\
\hline \multirow[t]{2}{*}{$2 \_80$} & 87.12 & 142.73 & 16.21 & 4 & 9 & 17 & 29 & 48 & 70 & 100 & 138 & 175 & 222 \\
\hline & & & & 4 & 11 & 20 & 36 & 58 & 81 & 112 & 146 & 189 & 240 \\
\hline \multirow[t]{2}{*}{$2 \_42$} & 104.27 & 164.99 & 17.94 & 4 & 8 & 14 & 21 & 32 & 48 & 67 & 91 & 117 & 149 \\
\hline & & & & 4 & 10 & 18 & 28 & 42 & 59 & 80 & 105 & 134 & 168 \\
\hline \multirow[t]{2}{*}{$2 \_4$} & 107.62 & 186.37 & 6.48 & 4 & 5 & 8 & 16 & 18 & 24 & 36 & 48 & 63 & 72 \\
\hline & & & & 4 & 8 & 10 & 15 & 22 & 26 & 38 & 54 & 64 & 80 \\
\hline \multirow[t]{2}{*}{$2 \_36$} & 166.29 & 262.64 & 12.30 & 4 & 8 & 13 & 20 & 29 & 41 & 56 & 72 & 89 & 110 \\
\hline & & & & 4 & 9 & 16 & 24 & 33 & 44 & 58 & 76 & 97 & 120 \\
\hline \multirow[t]{2}{*}{$2 \_69$} & 189.35 & 292.92 & 14.38 & 4 & 8 & 17 & 29 & 46 & 68 & 91 & 117 & 154 & 184 \\
\hline & & & & 4 & 9 & 17 & 28 & 49 & 69 & 92 & 119 & 151 & 184 \\
\hline
\end{tabular}

are known in the form of silicates, aluminosilicates or aluminophosphates, including those with low levels of heteroatom substitution, have $\vartheta<5$. This reflects the similarity of the preferred geometry between (alumino)silicates and AlPOs. The highest values of $\vartheta$ are 5.03 for AFY (Co-AlPO-50), which has $19 \%$ framework cobalt, and 5.18 for AHT, only known as the thermally unstable material AlPOH2. By analogy, we define structures with $\vartheta<5$ as feasible 'conventional' zeolites, i.e. those for which natural zeolites along with high-silica and AlPO forms are known. Framework types with

Structural figures were prepared using GDIS (SourceForge, 2004) and POV-Ray (Persistence of Vision Raytracer Pty. Ltd, 2004; Henson et al., 1994). The lattice energy, $\Delta E_{\text {quartz }}$, given in Table 1 , is relative to that of $\alpha$-quartz, calculated using the same potential model, and is thus analogous to the heat of transition reported for several high-silica zeolites (Henson et al., 1994; Petrovic et al., 1993; Navrotsky et al., 1995; Hu et al., 1995; Piccione et al., 2000, 2001, 2002; Moloy et al., 2002).

\subsection{The feasibility factor}

The well established relationship between framework density and calculated lattice energy (Foster et al., 2001, 2003; Foster, Friedrichs et al., 2004; Foster, Simperler et al., 2004; Simperler et al., 2004) was confirmed experimentally (Henson et al., 1994) for known zeolites. Using the standard leastsquares technique, a straight line was fitted to 145 data points obtained from minimizing quartz and all the known zeolite topologies in a purely siliceous form (Fig. 1). We excluded the four non-silicate structure types which substantially deviate from the rest: WEI (calcium beryllophosphate), CZP (sodium zincophosphate), OSO (potassium berylosilicate) and RWY (gallium germanium sulfide). The line of best fit has the formula $y=-1.4433 x+40.3904$, where $x$ is the framework

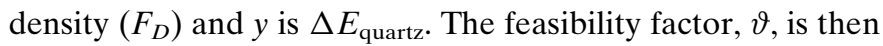
simply the dimensionless deviation of a data point $\left(x_{1}, y_{1}\right)$ from the line of best fit, given by the vertical offset $\vartheta=\left|1.4433 x_{1}+y_{1}+40.3904\right| / 1.4433$. Being formally independent of the framework density, the feasibility factor $\vartheta$ is thus a convenient way of discriminating between candidate structures and can be compared with the values obtained from known zeolite structures. We minimized all the known zeolite topologies as silica polymorphs, regardless of the actual composition in which they occur. We believe that $\vartheta$ is a better gauge of the feasibility of the structure than $\Delta E_{\text {quartz }}$ alone, as evidenced by the fact that seven of the ten lowest $\vartheta$ values in Table 1 belong to structures with known zeolite topologies. A ranking in order of ascending $\Delta E_{\text {quartz }}$ would, in contrast, produce only four values. Virtually all of the topologies which more 'exotic' compositions have $\vartheta>5$. For example, the zincosilicates VNI, VSV and RSN have $\vartheta$ of 5.75, 6.07 and 6.09, respectively. Beryllosilicates, generally containing three rings, also have higher $\vartheta$, e.g. LOV (6.51), NAB (10.99) and OSO (23.30), while the beryllophosphate weinebeneite has $\vartheta=$ 12.24 and the zincophosphate CZP $\vartheta=20.92$. We therefore propose that $\vartheta$ values up to 25 indicate that the topology may be feasible in the form of an 'oxide' material. Above this, we note that for RWY, the only zeotype structure known solely as a framework sulfide, $\vartheta=51.69$. Many other compositions, such as metal-organic frameworks, are of course possible. This means that although a structure may be deemed highly unfeasible as a zeolite, it may exist in other chemical forms. Also, the precise value of $\vartheta$ will be an unreliable guide in the high region, since it is based only on a silica model. In order to gauge the feasibility of a particular topology in a different composition, it would be necessary to carry out separate series of computations, taking into account the actual composition.

The Cerius ${ }^{2}$ software suite (Molecular Simulations Inc., 1999) was used for visualizing and manipulating the structures and for calculating free volumes, space-group symmetry and other parameters. In addition to calculating the energetics of the hypothetical structures, it is important to compare the calculated values with the values for all known zeolite frameworks. Thus, all relevant properties were also calculated for the purely siliceous forms of all known zeolite topologies. Lattice energies were calculated relative to $\alpha$-quartz, the most stable form of the mineral at ambient temperature.

The 'available volume', defined as the difference between the volume of the unit cell and the effective volume of all the atoms, depends on the van der Waals radius used for each atom. 'Occupiable volume' is the volume which can be occupied by a probe molecule with a given radius as it probes the surface of the structure. The 'accessible volume' is determined by tracing out the volume by the centre of the probe molecule as it follows the structure contours, but with the extra requirement that the probe must enter the unit cell from the outside via sufficiently wide pores or channels. The accessible volume gives an indication of the space available within each 
structure for applications in molecular sieving and catalysis. The calculations of the accessible volume were performed using the Free Volume module of the Cerius $^{2}$ package, which applies the Connolly (1985) method consisting of 'rolling' a probe molecule with a given radius over the van der Waals
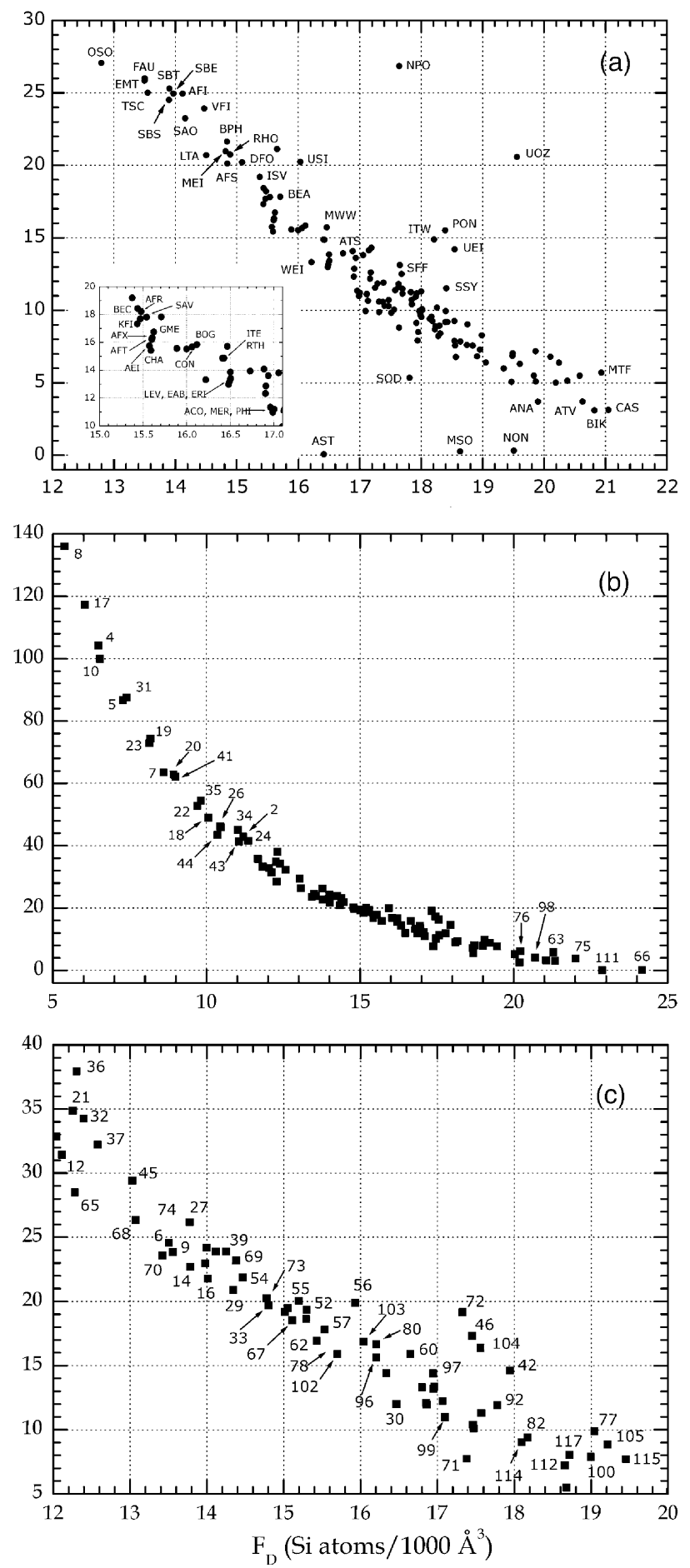

Figure 2

Accessible volume ( $\AA^{3}$ per Si atom) versus framework density for $(a)$ all known zeolitic structure types; (b) hypothetical binodal zeolitic structures; $(c)$ structures with accessible volumes below $40 \AA^{3}$ per $\mathrm{Si}$ atom. surface of the framework atoms. We have used a probe molecule with a radius of $1.4 \AA$ (such as water) and 1.32 and $0.9 \AA$ for the radii of $\mathrm{O}$ and $\mathrm{Si}$ atoms, respectively. The void volume, enclosed within the Connolly surface, was calculated first. The accessible volume was then calculated by requiring the probe molecule to enter the unit cell from the outside.

\section{Results and discussion}

Of the 117 structures, eight could not be optimized, either because refinement was not possible or because of failure during minimization, usually resulting in loss of the original network topology. The remaining 109 structures are described below. For the most part, these minimized smoothly without any loss of symmetry, although there are a few whose lowenergy symmetry is lower than that of the original space group. In these instances, the original space group is shown in parentheses in Table 2.

Figs. 1(a) and (b) show plots of framework energy relative to $\alpha$-quartz, $E_{F}$, versus the framework density, $F_{D}$, for all known zeolites. Relative framework energies of the hypothetical binodal frameworks range from $11.15 \mathrm{~kJ} \mathrm{~mol}^{-1}$ (structure 2_114) to as much as $515.43 \mathrm{~kJ} \mathrm{~mol}^{-1}$ (structure 2_72) (Fig. 1c). Fig. 1(d) plots the framework energy versus the framework density for the hypothetical binodal structures with energies below $30 \mathrm{~kJ} \mathrm{~mol}^{-1}$, the range considered as the most 'desirable', and with framework densities typical of the known zeolites.

Fig. 2(a) shows a plot of accessible volume versus framework density for the known structural types and Figs. 2(b) and (c) the corresponding plot for hypothetical binodal zeolites. Low framework density structures are of particular interest as they have very high accessible free volumes. Of the structures with framework densities below $18 \mathrm{Si}$ atoms $/ 1000 \AA^{3}$, structures 2_57, 2_58, 2_59, 2_82, 2_85, 2_86, 2_87, 2_91, 2_95, 2_96, 2_102, 2_103, 2_106, 2_108, 2_109, 2_110, 2_112, 2_113, 2_114 and 2_117 are energetically stable (Fig. 1c). Many hypothetical structures have dense frameworks, which are largely inaccessible. However, as many known zeolite topologies have low accessible volumes (Fig. 2a), a structure cannot be ruled out as a feasible topology on the basis of a low accessible free volume, even though it may be of no interest to scientists studying sorption, ion exchange or catalysis. A plot of framework density for known zeolites and for dense silicate frameworks against the size of the smallest ring in the structure (Brunner \& Meier, 1989) shows that very open frameworks with low $F_{D}$ have the largest number of four- and threemembered rings and that there is a gap in $F_{D}$ between compact minerals, such as quartz and tridymite, and the zeolite frameworks. The lower boundary of $F_{D}$ for known zeolites is from about 11 tetrahedral atoms per $1000 \AA^{3}$ in materials with four-membered rings to about 17 tetrahedral atoms in materials with $5+$ rings, where the plus sign signifies that some tetrahedral atoms are associated only with the larger rings.

Fig. 3 plots the framework energy with respect to $\alpha$-quartz for the known zeolitic structures and the hypothetical binodal structures versus the accessible volume, thus combining 
information contained in Figs. 1 and 2. Structures of the greatest practical interest are those with low energies and large volumes (see inset in Fig. 3b). Full details of all the structures have recently been published elsewhere (Foster, Simperler et al., 2004). Crystallographic CIF files from which powder X-ray diffraction patterns can be easily calculated are given as supplementary information. ${ }^{1}$

The structures have been divided into 15 families, the members of which share a common building scheme or structural unit. As explained above, the building units used do not necessarily equate to SBUs or PerBUs in the strict sense. We also note that the allocation of a structure to a certain family is not unequivocal: there are several structures which could equally well be assigned to more than one family. The order in which the various families are discussed is dictated by the feasibility factor of the most feasible structure in that family. Selected members of a particular family are shown in Figs. 4-9 in the same order, whereas a full description of all members is available in the electronic supplement. The more feasible structures will thus be encountered earlier in the following sections, with the exception of the 'orphan family' which contains several chemically feasible members. In describing the various structures, we use standard nomenclature from the zeolite literature. For instance, 'D6R' refers to a double six-ring unit. In describing polyhedral cages or units, the $\left[M^{x} N^{y}\right]$ system adopted by Smith (1988) is also used, where $(M, N)$ is the number of edges defining a given face and $(x, y)$ is the number of times that face appears in the polyhedron. Results are also tabulated in Table 1 (in order of $\vartheta$ ) and Table 2 (in numerical order of the structures). Table 1 gives $\vartheta, \Delta E_{\text {quartz }}$, the framework density and the coordination sequences of the $T$ sites. Table 2 gives the crystallographic data.

\subsection{ABC-6 family}

Of the 109 refinable binodal structures, 13 can be described using the building scheme for the ABC-6 family (van Koningsveld, 2004). Six of these are known frameworks: 2_89 = ERI, 2_84 = EAB, 2_90 = SAT, 2_83 = LEV, 2_107 = LOS and 2_78 = AFX. The PerBU of the family consists of a hexagonal array of isolated six-membered rings, which are related by pure translations along [100] and [010]. A three letter code $(A, B$ and $C)$ gives the connection mode of the layers along [001]. The six-membered rings of $A$ are centred at $(0,0)$, while layer $B$ is shifted by $(+2 / 3 a,+1 / 3 b)$ and layer $C$ by $(+1 / 3 a,+2 / 3 b)$. The connection between six-rings in adjacent layers is invariably via four-rings. In the (001) projection, there is a close similarity between all the structures of this family, epitomized by that of 2_106 (Fig. 4a), where the hexagonal array of six-rings, interspersed by four-rings, is clearly evident. Each structure is uniquely characterized by its [001] stacking sequence and the stacking sequences of the 13 structures (in order of their 'thermodynamic feasibility') are

\footnotetext{
${ }^{1}$ Supplementary data for this paper are available from the IUCr electronic archives (Reference: BK5018). Services for accessing these data are described at the back of the journal.
}

$A B B A C B B C(A)$ for 2_87, $A C A A B A(A)$ for 2_89 (ERI), $A C C A B B(A)$ for 2_84 $(E A B), A A B A B B C B C C A C(A)$ for 2_90 (SAT), $A B B C(A)$ for 2_86, $A A C B B A C C B(A)$ for 2_83 (LEV), $A B A C(A)$ for 2_107 (LOS), $A C A B A B C B C(A)$ for 2_110, $A C A B C B(A)$ for 2_106, $A C A C B A B A C B C B(A)$ for 2_108, $A C C A A B B A(A)$ for 2_78 (AFX), $A C C C B B B A A(A)$ for 2_40, and $A A A A C C C C B B B B(A)$ for 2_33. 2_87, 2_89, 2_84,2_107, 2_106 and 2_78, which have hexagonal symmetry, space group $P 6_{3} / m m c$, while 2_90, 2_83, 2_110, 2_108, 2_40 and 2_33 (all $R \overline{3} \mathrm{~m})$ and 2_86 $(P \overline{3} \mathrm{~m} 1)$ are trigonal. The ABC-6 structures, both known and hypothetical, are among the most thermodynamically favoured as silica polymorphs and, as can be seen from Table 1, have high chemical feasibilities $(0.08<\vartheta$ $<0.98$ ), except for 2_40 and 2_33 which have $\vartheta$ of 14.97 and 20.83 , respectively. The ABC-6 structures may also be thought of in terms of stacks, or chains, of cages linked parallel to the [001] direction through six-rings and, depending on symmetry, there are either one or two distinct types of stack. For example, the most feasible structure 2_87 (Figs. $4 b$ and $c$ ) contains both the $\left[4^{9} 6^{2} 8^{3}\right]$ gmelinite cages and $\left[4^{9} 6^{8} 8^{3}\right]$ EAB cages, which alternate along (001) (Fig. 4c). Parallel to these are stacks of alternating sodalite cages and double six-rings

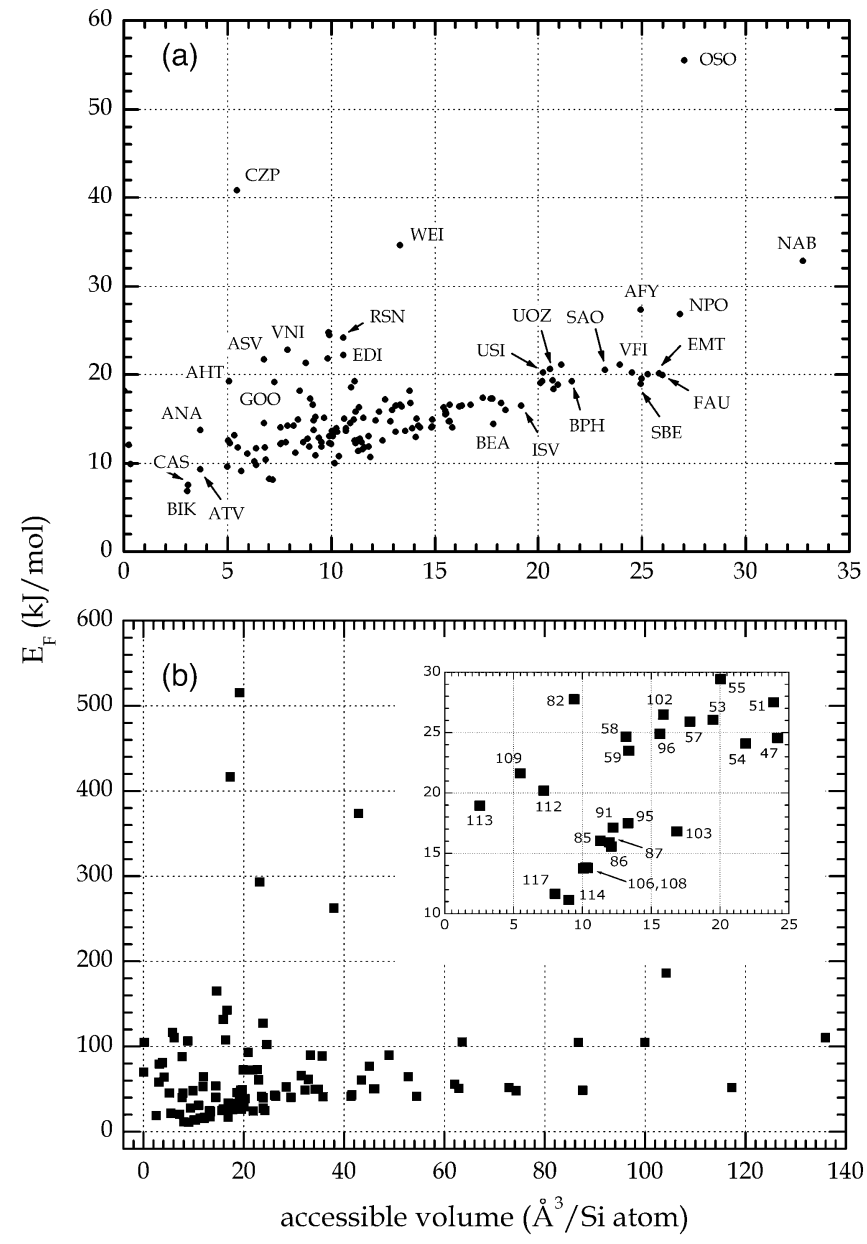

Figure 3

Framework energy with respect to $\alpha$-quartz versus accessible volume $\left(\AA^{3}\right.$ per $\mathrm{Si}$ atom) for $(a)$ all known zeolitic structure types; $(b)$ hypothetical binodal zeolitic structures. Hypothetical structures of particular chemical interest are identified in the inset. 
Table 2

Space groups and unit-cell dimensions of 109 hypothetical binodal zeolites, optimized as purely siliceous structures.

\begin{tabular}{|c|c|c|c|c|c|c|c|c|}
\hline Structure & $\begin{array}{l}\text { Space-group } \\
\text { symbol }\end{array}$ & $\begin{array}{l}\text { Space-group } \\
\text { number }\end{array}$ & $a(\AA)$ & $b(\AA)$ & $c(\AA)$ & $\alpha\left(^{\circ}\right)$ & $\beta\left(^{\circ}\right)$ & $\gamma\left({ }^{\circ}\right)$ \\
\hline $2 \_4$ & $\operatorname{Im} \overline{3} m$ & 229 & 24.5550 & 24.5550 & 24.5550 & 90 & 90 & 90 \\
\hline $2 \_5$ & $\operatorname{Im} \overline{3} m$ & 229 & 23.6252 & 23.6252 & 23.6252 & 90 & 90 & 90 \\
\hline $2 \_6$ & $P n \overline{3} m$ & 224 & 19.2265 & 19.2265 & 19.2265 & 90 & 90 & 90 \\
\hline $2 \_7$ & $P \overline{4} 3 m$ & 215 & 14.0784 & 14.0785 & 14.0785 & 90 & 90 & 90 \\
\hline $2 \_8$ & $\operatorname{Im} \overline{3} m$ & 229 & 29.9045 & 29.9046 & 29.9046 & 90 & 90 & 90 \\
\hline 2_9 & $R \overline{3} m$ & 166 & 20.6871 & 20.6871 & 10.7470 & 90 & 90 & 120 \\
\hline 2_10 & $\operatorname{Im} \overline{3} m$ & 229 & 30.8610 & 30.8610 & 30.8610 & 90 & 90 & 90 \\
\hline 2_12 & $I 4_{1} /$ amd & 141 & 15.1769 & 15.1769 & 17.2033 & 90 & 90 & 90 \\
\hline 2_13 & $\mathrm{Fm} / \mathrm{m}$ & 225 & 17.4521 & 17.4521 & 17.4521 & 90 & 90 & 90 \\
\hline 2_14 & $I 4 / \mathrm{mmm}$ & 139 & 11.5929 & 11.5929 & 12.9540 & 90 & 90 & 90 \\
\hline 2_15 & $P m / n$ & 223 & 13.6367 & 13.6367 & 13.6367 & 90 & 90 & 90 \\
\hline 2_16 & $\mathrm{P}_{2} / \mathrm{mnm}$ & 136 & 10.2171 & 10.2171 & 16.3964 & 90 & 90 & 90 \\
\hline 2_17 & $\operatorname{Im} \overline{3} m$ & 229 & 31.6666 & 31.6666 & 31.6666 & 90 & 90 & 90 \\
\hline 2_18 & $\operatorname{Im} \overline{3} m$ & 229 & 18.1332 & 18.1332 & 18.1332 & 90 & 90 & 90 \\
\hline 2_19 & $\operatorname{Im} \overline{3} m$ & 229 & 26.0211 & 26.0211 & 26.0211 & 90 & 90 & 90 \\
\hline 2_20 & $P m \overline{3} m$ & 221 & 17.5191 & 17.5191 & 17.5191 & 90 & 90 & 90 \\
\hline 2_21 & $F m \overline{3} m$ & 225 & 31.5256 & 31.5256 & 31.5256 & 90 & 90 & 90 \\
\hline 2_22 & $P m \overline{3} m$ & 221 & 19.5087 & 19.5087 & 19.5087 & 90 & 90 & 90 \\
\hline $2 \_23$ & $F m \overline{3} m$ & 225 & 32.8255 & 32.8255 & 32.8255 & 90 & 90 & 90 \\
\hline $2 \_24$ & $P m \overline{3} m$ & 221 & 14.6896 & 14.6986 & 14.6896 & 90 & 90 & 90 \\
\hline $2 \_25$ & $F m \overline{3} m$ & 225 & 30.1897 & 30.1897 & 30.1897 & 90 & 90 & 90 \\
\hline $2 \_26$ & $F m \overline{3} m$ & 225 & 30.2151 & 30.2151 & 30.2151 & 90 & 90 & 90 \\
\hline 2_27 & $F m \underline{3} \bar{m}$ & 225 & 27.5480 & 27.548 & 27.5480 & 90 & 90 & 90 \\
\hline 2_28 & $\operatorname{Im} \overline{3}$ & 204 & 16.0155 & 16.0155 & 16.0155 & 90 & 90 & 90 \\
\hline 2_29 & $F d \overline{3} c$ & 228 & 29.9172 & 29.9172 & 29.9172 & 90 & 90 & 90 \\
\hline $2 \_30$ & $\operatorname{Pn} \overline{3} m$ & 224 & 15.3879 & 15.3879 & 15.3879 & 90 & 90 & 90 \\
\hline 2_31 & $R \overline{3} m(F d \overline{3} m)$ & 166 (227) & 26.3028 & 26.3028 & 64.9314 & 90 & 90 & 120 \\
\hline $2 \_32$ & $\operatorname{Im} \overline{3} m$ & 229 & 24.9270 & 24.9270 & 24.9270 & 90 & 90 & 90 \\
\hline $2 \_33$ & $R \overline{3} m$ & 166 & 13.1741 & 13.1741 & 32.3570 & 90 & 90 & 120 \\
\hline $2 \_34$ & $\operatorname{Im} \overline{3} m$ & 229 & 25.9183 & 25.9183 & 25.9183 & 90 & 90 & 90 \\
\hline $2 \_35$ & $F d \overline{3} m$ & 227 & 30.8413 & 30.8416 & 30.8413 & 90 & 90 & 90 \\
\hline $2 \_36$ & $P m \overline{3} m$ & 221 & 19.8342 & 19.8342 & 19.8342 & 90 & 90 & 90 \\
\hline $2 \_37$ & $P n \overline{3} m$ & 224 & 19.6887 & 19.6887 & 19.6887 & 90 & 90 & 90 \\
\hline 2_39 & $P 1(\operatorname{Im} \overline{3} m)$ & 1 (229) & 21.5940 & 21.6260 & 21.6370 & 90.1262 & 89.9558 & 90.0775 \\
\hline $2 \_40$ & $R \overline{3} m$ & 166 & 13.2084 & 13.2084 & 23.8034 & 90 & 90 & 120 \\
\hline 2_41 & $\operatorname{Im} \overline{3} m$ & 229 & 25.2127 & 25.2127 & 25.2127 & 90 & 90 & 90 \\
\hline $2 \_42$ & $\operatorname{Im} \overline{3} m$ & 229 & 20.0209 & 20.0209 & 20.0209 & 90 & 90 & 90 \\
\hline $2 \_43$ & $F m \overline{3} m$ & 225 & 25.9040 & 25.9040 & 25.9040 & 90 & 90 & 90 \\
\hline $2 \_44$ & $P \overline{4} 3 m$ & 215 & 11.3123 & 11.3124 & 11.3124 & 90 & 90 & 90 \\
\hline $2 \_45$ & $P m \overline{3} m$ & 221 & 17.6786 & 17.6786 & 17.6786 & 90 & 90 & 90 \\
\hline $2 \_46$ & $P n \overline{3} m$ & 224 & 15.4919 & 15.4919 & 15.4919 & 90 & 90 & 90 \\
\hline $2 \_47$ & $P m \overline{3} m$ & 221 & 19.0003 & 19.0003 & 19.0003 & 90 & 90 & 90 \\
\hline $2 \_48$ & $P m \overline{3} n$ & 223 & 16.7613 & 16.7613 & 16.7613 & 90 & 90 & 90 \\
\hline $2 \_50$ & $P \overline{3} 1 m$ & 162 & 12.3351 & 12.3351 & 8.6007 & 90 & 90 & 120 \\
\hline $2 \_51$ & $\mathrm{P6}_{3} / \mathrm{mcm}$ & 193 & 12.3340 & 12.3340 & 17.2043 & 90 & 90 & 120 \\
\hline $2 \_52$ & $\operatorname{Pn} \overline{3} m$ & 224 & 16.7584 & 16.7584 & 16.7584 & 90 & 90 & 90 \\
\hline $2 \_53$ & $\operatorname{Pn} \overline{3} m$ & 224 & 18.5448 & 18.5448 & 18.5448 & 90 & 90 & 90 \\
\hline $2 \_54$ & $\mathrm{I} / \mathrm{mmm}$ & 139 & 14.8438 & 14.8438 & 20.0782 & 90 & 90 & 90 \\
\hline $2 \_55$ & $\mathrm{~Pb}_{3} / \mathrm{mcm}$ & 193 & 13.7562 & 13.7562 & 19.2727 & 90 & 90 & 120 \\
\hline $2 \_56$ & $P \overline{3} 1 m$ & 162 & 13.7003 & 13.7003 & 9.2686 & 90 & 90 & 120 \\
\hline $2 \_57$ & $I 4 / \mathrm{mmm}$ & 139 & 14.0993 & 14.0993 & 15.5435 & 90 & 90 & 90 \\
\hline $2 \_58$ & $I 4 / \mathrm{mmm}$ & 139 & 13.5265 & 13.5265 & 20.6385 & 90 & 90 & 90 \\
\hline $2 \_59$ & $\mathrm{P} 4 / \mathrm{nmm}$ & 129 & 13.5133 & 13.5133 & 10.3319 & 90 & 90 & 90 \\
\hline $2 \_60$ & $\operatorname{Im} \overline{3} m$ & 229 & 17.9320 & 17.9320 & 17.9320 & 90 & 90 & 90 \\
\hline 2_61 & $I 4_{1} /$ amd & 141 & 16.3875 & 16.3875 & 10.9441 & 90 & 90 & 90 \\
\hline $2 \_62$ & $P m \overline{3} m$ & 221 & 14.5985 & 14.5985 & 14.5985 & 90 & 90 & 90 \\
\hline $2 \_63$ & $I \overline{4} m 2$ & 119 & 12.6142 & 12.6142 & 9.4486 & 90 & 90 & 90 \\
\hline $2 \_64$ & $P m \overline{3} m$ & 221 & 18.3419 & 18.3419 & 18.3419 & 90 & 90 & 90 \\
\hline $2 \_65$ & $I a \overline{3}$ & 206 & 18.0311 & 18.0311 & 18.0311 & 90 & 90 & 90 \\
\hline $2 \_67$ & $\operatorname{Ia} \overline{3} d$ & 230 & 19.9520 & 19.9520 & 19.9520 & 90 & 90 & 90 \\
\hline $2 \_68$ & $I 4_{1} /$ amd & 141 & 15.1043 & 15.1043 & 10.7274 & 90 & 90 & 90 \\
\hline $2 \_69$ & $R \overline{3} m$ & 166 & 16.6853 & 16.6853 & 20.9554 & 90 & 90 & 120 \\
\hline $2 \_70$ & $P m \overline{3} m$ & 221 & 13.8940 & 13.8940 & 13.8940 & 90 & 90 & 90 \\
\hline 2_71 & $P 2_{1} 3$ & 198 & 14.0298 & 14.0298 & 14.0298 & 90 & 90 & 90 \\
\hline $2 \_73$ & $F d \stackrel{\frac{1}{3}}{m}$ & 227 & 29.6184 & 29.6184 & 29.6184 & 90 & 90 & 90 \\
\hline $2 \_74$ & $F m \overline{3} m$ & 225 & 30.4872 & 30.4872 & 30.4872 & 90 & 90 & 90 \\
\hline 2_75 & $F d \overline{3} m$ & 227 & 25.9368 & 25.9368 & 25.9368 & 90 & 90 & 90 \\
\hline $2 \_76$ & $P \overline{4} 3 m$ & 215 & 13.3418 & 13.3418 & 13.3418 & 90 & 90 & 90 \\
\hline $2 \_77$ & $P n \overline{3} m$ & 224 & 15.5787 & 15.5787 & 15.5787 & 90 & 90 & 90 \\
\hline
\end{tabular}

(D6R). The structure 2_40, which is less dense, is quite interesting as it has large cages linked through elongated 10- and 12-rings, respectively (Fig. $4 d$ and $e$ ).

\section{2. $\left[3^{2} 5^{6}\right]$ family}

Four structures (2_103, 2_55, 2_56 and 2_104) are built up from columns of $\left[3^{2} 5^{6}\right]$ polyhedral units (Fig. $4 g$ ) arranged hexagonally so as to give 12 membered ring channels along the $c$ direction (Fig. 4f). The $\left[3^{2} 5^{6}\right]$ units are linked by sharing their 'terminal' three-membered ring windows (Fig. $4 h)$ in structures 2_103 and 2_104, while in structures 2_55 and 2_56 these small cage units are separated by a $\left[3^{2} 4^{3}\right]$ unit (i.e. a trigonal prism or D3R; Fig. 4i). None of these four are known structures, although 2_103 is expected to be highly chemically feasible $(\vartheta=0.30)$. Three further members of this family, 2_112, 2_102 and 2_80, also contain the $\left[3^{2} 5^{6}\right]$ unit (Fig. $4 g$ ), but with different building patterns. For example, in 2_112 the $\left[3^{2} 5^{6}\right]$ units are linked via single oxygen bridges, while in 2_102 and $2 \_80$ the units are linked via double oxygen bridges (Fig. 4j). Both 2_112 and 2_102 are highly feasible, with $\vartheta=$ 4.66 and 6.08 , respectively, as opposed to $2 \_80$ which has $\vartheta=87.12$.

\subsection{AWW family}

The nine structures which we describe as members of the "AWW family' share a small $\left[4^{6} 6^{4}\right]$ cage as the common building unit (Fig. $5 a$ ). Six of these structures, 2_88 (which has the actual AWW topology), 2_85, 2_59, 2_58, 2_100 and 2_63, are tetragonal, with columns of larger cages parallel to [001] and having eight-ring windows as the maximum pore diameter in that direction. The archetypal example is the AWW $\left[4^{8} 6^{8} 8^{2}\right]$ cage (Fig. $5 b$ ), which stacks through shared eight-rings. Fig. 5(c) shows the [001] projection of 2_85, which is typical of this series.

Depending on the linkage pattern of the $\left[4^{6} 6^{4}\right]$ building units along [001], different types of large cage are defined. AWW, 2_59, 2_100 and 2_63 
Table 2 (continued)

\begin{tabular}{|c|c|c|c|c|c|c|c|c|}
\hline Structure & $\begin{array}{l}\text { Space-group } \\
\text { symbol }\end{array}$ & $\begin{array}{l}\text { Space-group } \\
\text { number }\end{array}$ & $a(\AA)$ & $b(\AA)$ & $c(\AA)$ & $\alpha\left({ }^{\circ}\right)$ & $\beta\left(^{\circ}\right)$ & $\gamma\left({ }^{\circ}\right)$ \\
\hline $2 \_78$ & $P 6_{3} / m m c$ & 194 & 13.5479 & 13.5479 & 19.3503 & 90 & 90 & 120 \\
\hline $2 \_79$ & $\operatorname{Im} \overline{3} m$ & 229 & 21.2424 & 21.2424 & 21.2424 & 90 & 90 & 90 \\
\hline $2 \_80$ & $\operatorname{Im} \overline{3} m$ & 229 & 18.0938 & 18.0938 & 18.0938 & 90 & 90 & 90 \\
\hline $2 \_81$ & $\mathrm{I} 4 / \mathrm{mmm}$ & 139 & 13.9993 & 13.9993 & 10.2051 & 90 & 90 & 90 \\
\hline $2 \_82$ & $\operatorname{Pn} \overline{3} m$ & 224 & 15.8240 & 15.8240 & 15.8240 & 90 & 90 & 90 \\
\hline $2 \_83$ & $R \overline{3} m$ & 166 & 12.9786 & 12.9786 & 22.4610 & 90 & 90 & 120 \\
\hline $2 \_84$ & $P 6_{3} / m m c$ & 194 & 12.9887 & 12.9887 & 14.9436 & 90 & 90 & 120 \\
\hline $2 \_85$ & $I 4 / \mathrm{mmm}$ & 139 & 13.2812 & 13.2812 & 15.4875 & 90 & 90 & 90 \\
\hline $2 \_86$ & $P \overline{3} m 1$ & 164 & 12.7931 & 12.7931 & 10.0490 & 90 & 90 & 120 \\
\hline $2 \_87$ & $P 6_{3} / m m c$ & 194 & 12.7982 & 12.7982 & 20.0706 & 90 & 90 & 120 \\
\hline 2_88 & $P 4 / n m m$ & 129 & 13.5200 & 13.5199 & 7.6115 & 90 & 90 & 90 \\
\hline $2 \_89$ & $P 6_{3} / m m c$ & 194 & 12.9122 & 12.9122 & 15.1051 & 90 & 90 & 120 \\
\hline 2_90 & $R \overline{3} m$ & 166 & 12.7260 & 12.7259 & 30.3678 & 90 & 90 & 120 \\
\hline 2_91 & $\mathrm{I} / \mathrm{mcm}$ & 140 & 13.9768 & 13.9768 & 19.1953 & 90 & 90 & 90 \\
\hline 2_92 & $\mathrm{P} 4 / \mathrm{nbm}$ & 125 & 13.9490 & 13.9490 & 9.2497 & 90 & 90 & 90 \\
\hline 2_93 & $\operatorname{Im} 3 m$ & 229 & 17.2697 & 17.2697 & 17.2697 & 90 & 90 & 90 \\
\hline 2_94 & $C 2(F d \overline{3} m)$ & 5 (227) & 29.4382 & 29.3841 & 20.7989 & 90 & 90 & 90 \\
\hline 2_95 & $I 4 / \mathrm{mmm}$ & 139 & 12.2058 & 12.2058 & 19.1794 & 90 & 90 & 90 \\
\hline 2_96 & $\operatorname{Im} \overline{3}$ & 204 & 16.4413 & 16.4413 & 16.4413 & 90 & 90 & 90 \\
\hline 2_97 & $P m 3 n$ & 223 & 16.1973 & 16.1973 & 16.1973 & 90 & 90 & 90 \\
\hline 2_98 & $P 4_{1} 32$ & 213 & 11.5642 & 11.5642 & 11.5642 & 90 & 90 & 90 \\
\hline 2_99 & $P m 3$ & 200 & 12.8171 & 12.8171 & 12.8171 & 90 & 90 & 90 \\
\hline $2 \_100$ & $I \overline{4} m 2$ & 119 & 12.8690 & 12.8691 & 7.6292 & 90 & 90 & 90 \\
\hline 2_101 & $F m 3 m$ & 225 & 13.4592 & 13.4592 & 13.4592 & 90 & 90 & 90 \\
\hline 2_102 & $R \overline{3} m$ & 166 & 12.6141 & 12.6141 & 16.6417 & 90 & 90 & 120 \\
\hline 2_103 & $\mathrm{Pb}_{3} / \mathrm{mcm}$ & 193 & 13.6152 & 13.6152 & 13.9813 & 90 & 90 & 120 \\
\hline 2_104 & $P \overline{3} 1 m_{-}$ & 162 & 13.4810 & 13.4810 & 6.5129 & 90 & 90 & 120 \\
\hline 2_105 & $P 1(R \overline{3} c)$ & 1 (167) & 10.6747 & 16.8789 & 16.9018 & 67.8079 & 86.0781 & 86.1532 \\
\hline 2_106 & $P 6_{3} / m m c$ & 194 & 12.4093 & 12.4093 & 15.4571 & 90 & 90 & 120 \\
\hline 2_107 & $P 6_{3} / m m c$ & 194 & 12.3972 & 12.3972 & 10.3205 & 90 & 90 & 120 \\
\hline 2_108 & $R \overline{3} m$ & 166 & 12.4186 & 12.4186 & 30.8573 & 90 & 90 & 120 \\
\hline 2_109 & $P n \overline{3} m$ & 224 & 17.2562 & 17.2562 & 17.2562 & 90 & 90 & 90 \\
\hline 2_110 & $R \overline{3} m$ & 166 & 12.4060 & 12.4060 & 23.1948 & 90 & 90 & 120 \\
\hline 2_111 & $P 4_{1} 32$ & 213 & 11.6324 & 11.6324 & 11.6324 & 90 & 90 & 90 \\
\hline 2_112 & $P 2_{1} 3$ & 198 & 13.7019 & 13.7019 & 13.7019 & 90 & 90 & 90 \\
\hline 2_113 & Fddd & 70 & 7.4170 & 13.5469 & 23.6645 & 90 & 90 & 90 \\
\hline 2_114 & $\mathrm{I} / \mathrm{mcm}$ & 140 & 13.7055 & 13.7055 & 14.1225 & 90 & 90 & 90 \\
\hline 2_115 & $P 4 / n b m$ & 125 & 13.4128 & 13.4128 & 6.8567 & 90 & 90 & 90 \\
\hline 2_116 & $I 432$ & 211 & 16.4510 & 16.4519 & 16.4510 & 90 & 90 & 90 \\
\hline $2 \_117$ & $\mathrm{P}_{2} / \mathrm{mnm}$ & 136 & 7.1839 & 7.1839 & 12.4079 & 90 & 90 & 90 \\
\hline
\end{tabular}

[100] view of 2_45 (Fig. 5e). Structure $2 \_74$ has the framework of the mineral tschörtnerite (TSC) with both sodalite and alpha cages linked via D6R (Fig. 5f), thus defining the large TSC cage (Fig. $5 g$ ). The remaining structures will be discussed with respect to structural similarities and not by their chemical feasibility factor, $\vartheta$.

Structures 2_35 (Fig. 5h) and 2_31 are composed of sodalite cages linked tetrahedrally via D6R and thus form a series together with the FAU structure. 2_35 and 2_31 are both feasible as oxide materials, with $\vartheta$ of 10.69 and 13.16, respectively. 2_45 (Fig. 5i) and 2_36 can similarly be imagined as belonging to a series with $\mathrm{RHO}$, a structure formed by alpha cages linked octahedrally via D8R. Both have $\operatorname{Pm} \overline{3} m$ symmetry and $2 \_45$ is relatively feasible $(\vartheta=12.91) .2 \_24$ (Fig. $5 j$ ) and 2_20 are related to the LTA structure, since they can be generated by linking sodalite cages and D4R. They also have the same supercages as 2_45 and 2_36 and similar $\vartheta$ of 13.28 and 16.11 , respectively. Structures 2_27 (Fig. $7 k$ ) and 2_21 can also be considered part of a series with LTA, except in this case it is the alpha cages which are retained and the linkages between them expanded. The final pair, 2_39 (Fig. $5 l$ ) and 2_32, form a series derived from KFI, containing alpha cages

have only one type of eight-ring channel cage each, whilst in 2_85 two alternating types of larger cage are thus defined, $\left[4^{8} 6^{12} 8^{2}\right]$ and $\left[4^{8} 6^{4} 8^{2}\right]$ (also found in the structures SAS and ATN respectively). Structures AWW, 2_58, 2_59 and 2_85 fall within the feasible range, with $\vartheta=0.32-6.04$, while 2_100 $(\vartheta=$ 22.42) and 2_63 $(\vartheta=73.83)$ are less feasible.

There are also three cubic structures, which contain the same building unit (2_109, 2_97 and 2_60), with 2_109 being by far the most feasible of the three $(\vartheta=5.67)$. For these three structures, the $\left[4^{6} 6^{4}\right]$ units alternate with sodalite or beta cages in a chain along [100]. Structure 2_97 $(\vartheta=16.99)$ falls within the extended range of oxide feasibility, whereas 2_60 $(\vartheta=$ 80.04) does not.

\subsection{Supercage family}

There are 11 structures which contain sodalite or LTA (alpha) cages linked by smaller prismatic units in such a way that it also generates much larger cages. All the structures have cubic or pseudo-cubic symmetry, as can be seen in the which are connected via shared D6R and are replaced by stacks of two and three D6R.

\subsection{SAS family}

These structures are analogous to the AWW family as they contain stacks of large cages linked unidirectionally by eightrings. Fig. 6(a) shows the [001] projection of structure 2_54, typical of all four tetragonal structures belonging to this family and having $I 4 / \mathrm{mmm}$ space-group symmetry [2_54, 2_57, 2_81 (SAS) and 2_95]. The basic building units may be thought of as smaller polyhedra arranged in parallel chains: in the case of 2_81 the basic units are D6R hexagonal prisms, which form a chain by sharing four-rings, 2_95 is a highly feasible $(\vartheta=0.93)$ structure in which $\left[4^{4} 5^{4}\right]$ units are linked into chains via fourrings (Fig. 6b); in 2_57 an additional D4R is interposed between the alternating D6R and 2_54 is built analogously from chains of alternating D8R and D4R. Aside from SAS and 2_95, both 2_54 and 2_57 are also quite feasible as zeolites $(\vartheta=3.18$ and 5.51, respectively). 


\section{6. $\left[4^{2} 5^{8}\right]$ family}

These structures have a small $\left[4^{2} 5^{8}\right]$ cage as the building unit (Fig. 6d). In four of the structures, these units are linked into chains through the four-rings which cap the cages. The structures are tetragonal with $\left[4^{2} 5^{8}\right]$ chains running along [001] and have large cages accessible through eight-rings. The projection of 2_91 along [001] is typical of this family (Fig. 6c). Structure 2_91 is the most feasible of these structures $(\vartheta=0.95)$ and has $\left[4^{2} 5^{8}\right]$ cages linked through D4R, with a chain repeat motif of two cages and two D4R. In 2_114, another highly feasible structure with $\vartheta=2.17$, the cages are directly linked through a shared four-ring. Structures 2_92 and 2_115 are analogous to structures 2_91 and 2_114, respectively, but with only half the chain repeat distance. Both structures are far less feasible, as is a fifth structure, 2_116 (Figs. $6 e$ and $f$ ), in which the $\left[4^{2} 5^{8}\right]$ units are linked into chains via pairs of $T-\mathrm{O}-T$ linkages (Fig. $6 f$ ). In the latter, the chains are interconnected so as to run in all three directions of the cubic lattice and the structure also contains sodalite cages, each of which shares its four-ring windows with $\left[4^{2} 5^{8}\right]$ units.

\subsection{AST family}

Structure 2_101 (Fig. $6 g-i$ ) is topologically identical to the known zeolite AST (AlPO-16). ${ }^{33,34}$ The structure contains the characteristic $\left[4^{6} 6^{10}\right]$ cages (Fig. 6i), but may also be thought of in terms of $\mathrm{D} 4 \mathrm{R}$ units connected through $\mathrm{O}-T-\mathrm{O}$ bridges

(Fig. 6h). In 2_73 the D4R connect through single oxygen bridges and, apart from containing sodalite cages, the structure also possesses large tetrahedral cages with 12-ring apertures. Structure 2_61 is tetragonal containing cages with ovalshaped ten-rings as their largest apertures. Topologically, 2_13 (Figs. $6 j$ and $l$ ) is a variation of the AST structure in which those $T$ atoms which do not form part of D4R are replaced by the $\left[3^{4}\right]$ tetrahedra of $T$ sites, a structural feature not found in aluminosilicate zeolites, although present, for instance, in the zeotypic sulfide RWY.

\subsection{D8R family}

This family is formed by four structures which contain the double eight-ring (D8R) as a structural unit. Structure 2_47 has a cubic structure in which the building unit may be thought of as a D8R with four D4R attached to alternate four-ring faces (Fig. 7a). The units do not link directly to one another, but are arranged so as to define the large $\left[4^{24} 6^{8} 8^{18}\right]$ (TSC) cages (Fig. 7b). Structures 2_19 and 2_17 form part of a homologous series of structures, together with the uninodal structure 1_11 (Foster et al., 2003), one of the nine simple uninodal tilings. The latter structure has a body-centered cubic framework based on chains of D8R and D4R, and 2_19 has the same structure, except that the D4R in 1_11 are replaced in 2_19 by pairs of face-sharing D4R (Fig. 7c) and

Figure 4

Molecular graphic illustrations of some structures from the ABC- 6 and $\left[3^{2} 5^{6}\right]$ families.

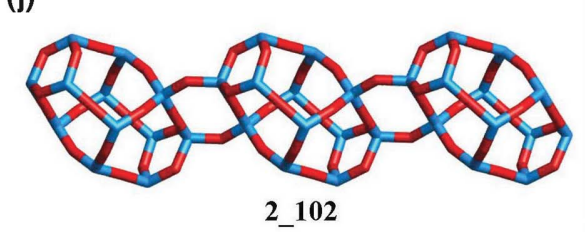


in 2_17 by groups of three D4R. The more complex 2_34 structure also contains the D8R/D4R units, but with the addition of $\left[4^{6} 6^{4}\right]$ AWW cages forming large cages with 12rings as the maximum aperture (Fig. $7 d$ ). Topologically, the tile which corresponds to this cage is the largest among this set of binodal frameworks, with 74 faces, 144 vertices and 216 edges. Structure 2_34 shares the space group $\operatorname{Im} \overline{3} m$ with both $2 \_19$ and 2_17. Structure 2_47 is thermodynamically feasible $(\vartheta=$ 3.02), while 2_17 and 2_19 have $\vartheta=13.96$ and 13.39 , respectively, despite having extremely low framework densities of 8.17 and $6.05 T$ per $1000 \AA^{3}$, respectively.

\subsection{AFY family}

Structure 2_50 is topologically identical to the known structural type AFY (AIPO-50). The secondary building unit of this family is a D4R, which in AFY form hexagonal layers (Fig. 7e) and are tilted with respect to the (001) plane. These layers then repeat through simple translation along $c$, most clearly seen in the (120) projection (Fig. 7f; van Koningsveld, 2004; Baelocher \& McCusker, 2004). If, instead, the layers alternate in orientation by means of a mirror plane (i.e. $A B A$ rather than $A A$ ), the hypothetical framework 2_51 is formed. Both have low $\vartheta$ values: 5.03 and 5.18 for 2_50 and 2_51, respectively, making 2_51 virtually as feasible as AFY.

\subsection{D6R family}

This family comprises seven structures $\left(2 \_6,2 \_30,2 \_53\right.$, 2_75, 2_76, 2_77 and 2_82) which have in common D6R hexagonal prisms as building units. These structures are all cubic, space group $P n \overline{3} m$, with the exception of 2_76 and 2_75, for which D6R (i.e. 6-6) may be strictly defined as a secondary building unit. The first five members of the group may be thought of in terms of chains running along [110] in which the D6R are linked by various combinations of rings. In the most feasible member of the family, 2_53 $(\vartheta=5.11)$, the link unit includes D4R, giving rise to the characteristic motif shown in Fig. $7(\mathrm{~g})$, where four D6R are connected to a single D4R. This structure also contains FAU supercages linked via the $\left[4^{18} 8^{6} 12^{2}\right]$ cages (Fig. $7 h$ ). Structure 2_82 is quite similar to 2_53, whereas in 2_77 the D6R chains are linked by units of three four-rings and in 2_30 a spiro-5 unit links the D6R into chains. Structure 2_6 also contains three-rings linked into $\left[3^{4}\right]$ tetrahedra which connect the D6R. Finally, structures 2_75 and 2_76 are the 'odd ones' of the family since it is not possible to describe them using the D6R chain model. Structure 2_75 is very unusual as it contains both 'regular' and flattened sodalite cages connected through six-rings (Fig. 7i). Structure 2_76 contains (differently) distorted beta cages as well as larger cages accessible through both approximately planar six-rings (a)

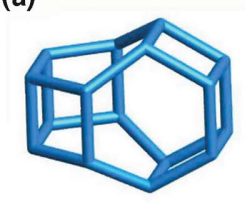

(b)

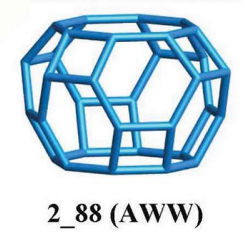

(c)

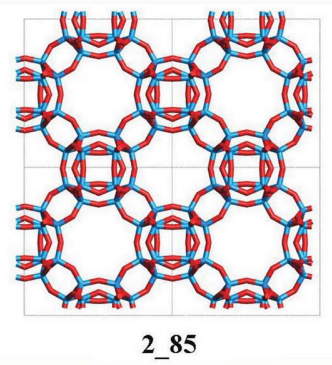

(d)

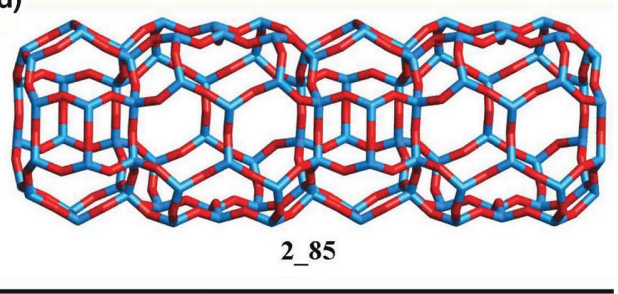

(e)

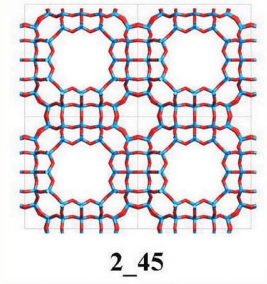

(i)

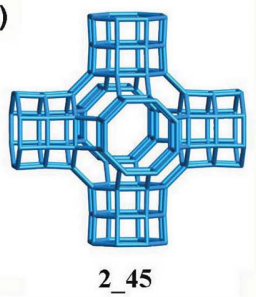

(f)

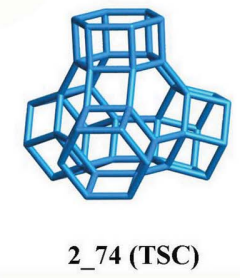

(j)

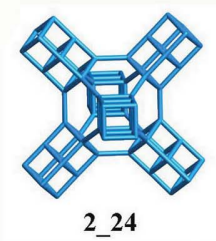

(g)

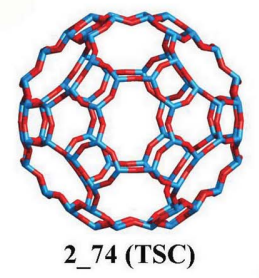

(k)

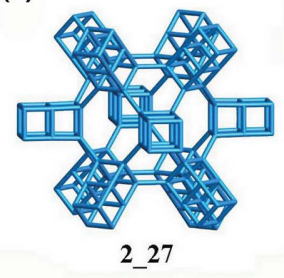

(h)

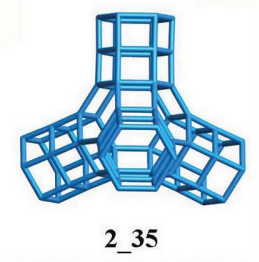

(I)

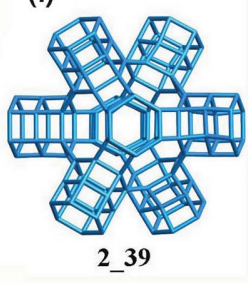

Figure 5

Molecular graphic illustrations of some structures from the AWW and supercage families. 
and highly curved eight-rings. Structures 2_75, 2_6 or 2_76 are not expected to be chemically feasible.

\subsection{Three- and four-ring family}

These eight structures are grouped together because they contain both three- and four-membered rings, although in other ways they are fairly different. Seven structures are cubic and five have framework densities lower than $14 T$ per $1000 \AA^{3}$. Structure 2_99, the most feasible structure of this family with $\vartheta=10.53$, can be described as a network of cornersharing three- and four-rings, part of which is the unit shown in Fig. 8(a). Three types of cages are found, one of which is the $\left[3^{8} 8^{6}\right]$ (truncated cube, Fig. $8 b$ ). The somewhat similar 2_62, which has $\vartheta=10.97$, also exhibits the truncated cube cage. Structure 2_68 has a low framework density of $13.08 \mathrm{~T}$ per $1000 \AA^{3}$ and $\vartheta=$ 13.82. Structures 2_70 and 2_93 have similar framework densities to that of 2_68 (13.42 and 13.98, respectively) and $\vartheta=14.33$ and 28.25 , making these three structures interesting candidates as zeotypes. Structure 2_93 contains $\left[3^{4} 6^{4}\right]$ cages, i.e. truncated tetrahedra (Fig. 8c), which link through shared three-rings to form a bodycentred cubic structure. Structures 2_18, 2_28 and 2_5 have much lower framework densities (10.06, 11.69 and 7.28 , respectively) than conventional zeolites, and are thus much less feasible as zeotype materials.

\subsection{2. $\left[3^{2} 4^{3}\right]$ D3R family}

The common feature is a trigonal prism (a $\left[3^{2} 4^{3}\right]$ unit) and we have assigned nine structures to this family. As in the previous family, many are of interest due to their low density, with the presence of small polyhedra being compensated by large supercages. While we believe that none is feasible in a traditional zeolite or AIPO composition, they may be of interest in several areas of chemistry, for instance if it were possible to form the D3R unit as a precursor. All the structures are cubic and have at least $m \overline{3} m$ symmetry. Structure 2_43 (Figs. $8 d-$ $f)$ is the most feasible $(\vartheta=11.62)$ and has D3R units attached to $\left[3^{4} 6^{4}\right]$ truncated tetrahedra to form tetrahedral units (Fig. 8e). 'Truncated cube' cages are present, as are the large $\left[4^{24} 6^{8} 8^{18}\right]$ cages shown in Fig. 8(f). In 2_64 the D3R are also attached to truncated cube cages, but the structure additionally

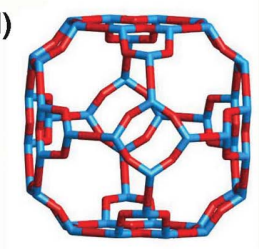

Figure 6

Molecular graphic illustrations of structures from the SAS, $\left[4^{2} 5^{8}\right]$ and AST families. 
contains alpha and $\left[4^{24} 6^{8} 8^{18}\right]$ tschörtnerite (TSC) cages. Structure 2_23 has beta cages linked via D3R-four-ring-D3R bridging units and 2_26 also has the same unit of two D3R linked though a four-ring (as do 2_25 and 2_22), with alpha cages present. Structure 2_25 has a pore system connected through 12-ring apertures and contains, besides FAU supercages and LTA alpha cages, the large $\left[4^{24} 8^{6} 12^{8}\right]$ cages found in the RWY structure. Structure 2_41 is similar to 2_43, as the
D3R form an alternating network with truncated tetrahedra (as in Fig. 8e). Structure 2_22 is also of very low density $\left(F_{D}=\right.$ $9.70 T$ per $1000 \AA^{3}$ ) and has the D3R connected so as to define D8R. Finally, 2_4 and 2_8 are among the least dense of all the binodal simple tile structures, with $F_{D}$ of 5.39 and $6.48 T$ per $1000 \AA^{3}$, respectively. The basic building unit of $2 \_8$ is two D3R stacked with an intervening D4R (Fig. 8g). In 2_4 the intermediate unit is absent and D3R units join directly through a shared four-ring. In both cases, very open cavity systems are constructed by connection of these units (Fig. 8h).

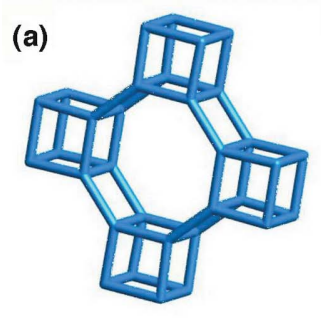

2_47 (b)

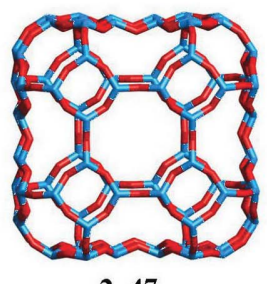

2_47 (c)

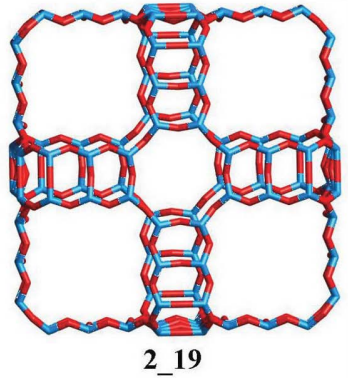

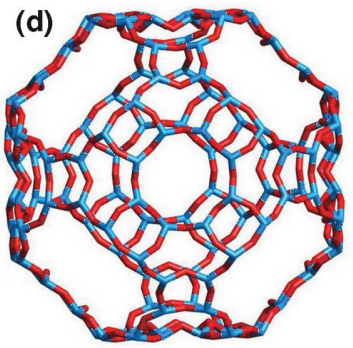

2_34 (e)

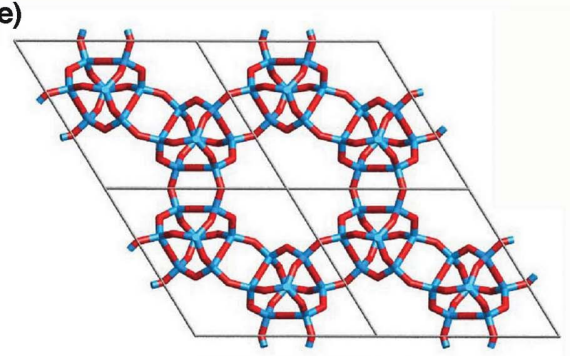

2_50 (AFY) (f)

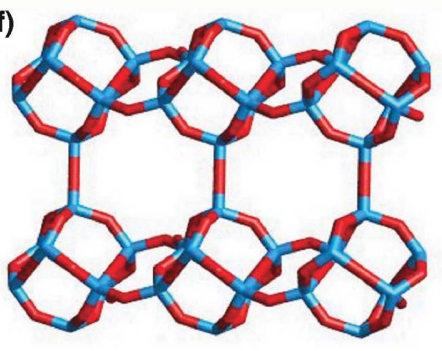

2_50 (AFY) (g)

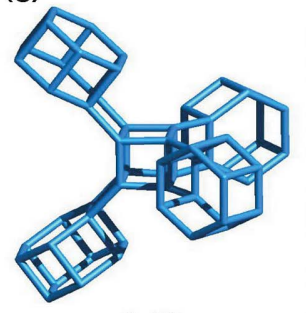

2_53

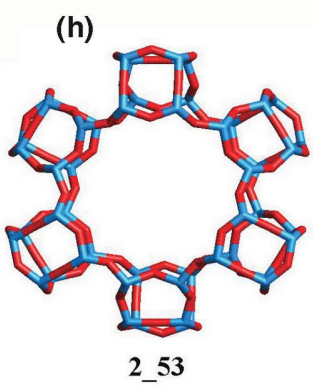

(i)

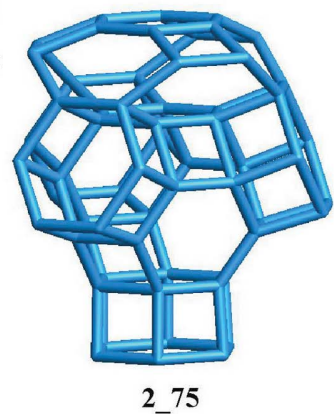

Figure 7

Molecular graphic illustrations of some structures from the D8R family, AFY structures and the D6R family.

\subsection{Three-ring family}

This family of eight structures is characterized by the presence of three-rings. Five structures contain pairs, or longer chains, of threerings which share one $T$ atom and therefore contain the spiro-5 unit (Baerlocher et al., 2001). Two of the structures also contain four-rings. As expected, several of the structures are of low density, but none would be expected to be realisable as a conventional zeolite. In 2_71, the most feasible with $\vartheta=17.07$, three-rings themselves form rings of six (Fig. 8i), with the structure also containing elongated cages having eight-rings as their largest pore. The basic unit of 2_69 is a pair of edgesharing three-rings (or bridged four-ring, Fig. 8j). These larger units then connect to define a hexagonal channel system. Structure 2_65 also contains loops of six three-rings, virtually identical in structure to those in 2_71. However, the structure is much more open $\left(F_{D}=12.28\right.$, compared with 17.38 for 2_71), containing a three-dimensional network of 10- and 12-ring pores. Structure 2_44 is another very open structure $\left(F_{D}=10.36\right)$, with a threedimensional network of cornersharing three-rings defining the small $\left[3^{4} 6^{4}\right]$ cages shown in Fig. $8(k)$, as well as large cavities linked through 12-rings. Structure 2_12 has unusual chains built up from pairs of edge-sharing three-rings and has large cross-linked channels extending in two dimensions, delineated by puckered 14-membered rings (Fig. 8l). Structure 2_29 is an 
unusually complex cubic structure, with three- and four-rings linked together (Fig. $8 \mathrm{~m}$ ): pairs of edge-sharing three-rings are formed (there are no spiro-5 units) and these pairs are further connected by distorted four-rings. Uniquely for this family, in 2_105 the three-rings do not directly link into chains or pairs through the sharing of $T$ atoms, but rather connect through oxygen bridges to define five-rings. Finally, 2_9 has H-shaped building units in which four-rings share edges with pairs of three-rings (Fig. $8 n$ ).

\subsection{4. $\left[3^{4}\right]$ family}

The common feature of this family is a $\left[3^{4}\right]$ unit, sometimes known as the 'supertetrahedron' or 'tetrahedron of tetrahedra'. This unit is unknown in zeolitic oxide materials, but is present in some sulfides, including the zeotypic RWY structure and the compound $\mathrm{Na}_{2} \mathrm{Si}_{2} \mathrm{~S}_{5}$. Structure 2_16 (Figs. 9a and $b$ ), one of the few structures containing seven-rings, is characterized by its $\left[3^{8} 4^{2} 7^{8} 8^{4}\right]$ cage (Fig. $9 b$ ). Each of the eight three-rings forms part of a $\left[3^{4}\right]$ unit, shared with three other cages. This structure is the most feasible of this family, with $\vartheta=36.19$. (a)

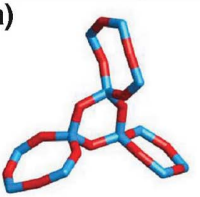

2_99

(d)

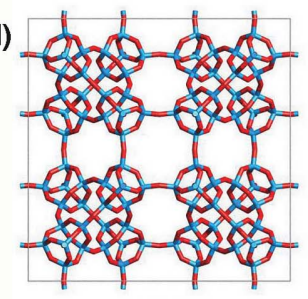

(g)

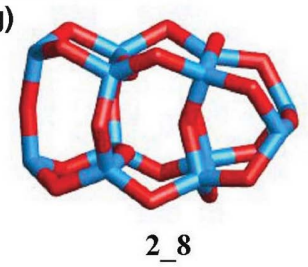

(b)

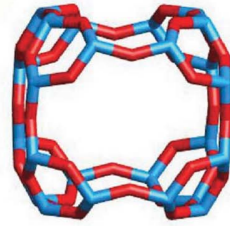

2_99 (c)

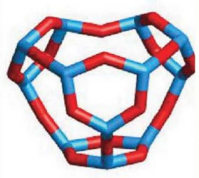

2_93

(e)

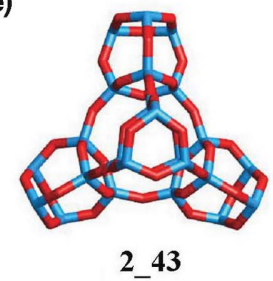

(f)

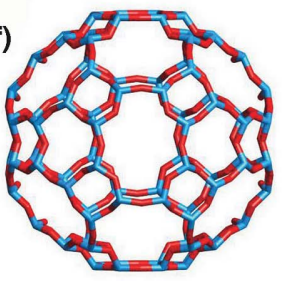

(h)

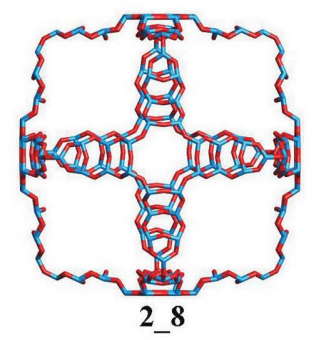

(i)

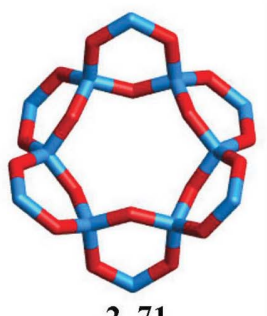

2_71

(I)

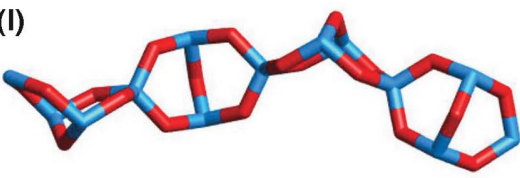

2_12 (j)

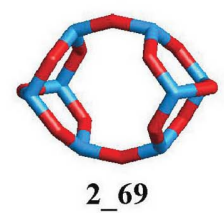

(k)

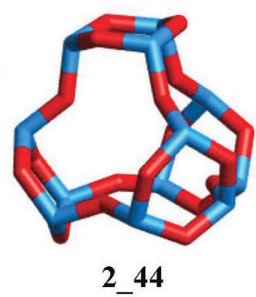

(n)

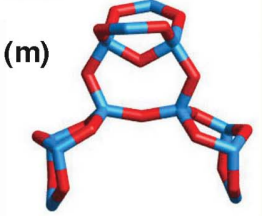

2_29

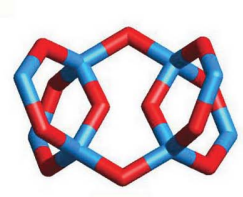

2_9

Figure 8

Molecular graphic illustrations of structures from the three- and four-ring family, the D3R family and the three-ring family.

Similarly, 2_14 has only one type of 'larger' cage, $\left[3^{8} 4^{2} 6^{4} 8^{8}\right]$, and the whole structure can be thought of in terms of the sodalite framework, but with one third of the $T$ sites replaced by $\left[3^{4}\right]$ supertetrahedra. Structure 2_15 is also related to the sodalite structure, although now with half of the original $T$ sites replaced by the $\left[3^{4}\right]$ units, creating $\left[3^{12} 6^{6} 9^{8}\right]$ cages. Structure 2_10 can be derived from the RHO zeolite structure by replacement of all $T$ sites by $\left[3^{4}\right]$ tetrahedra. As a result, it possesses very large cages linked via double 16-membered rings (Fig. 9c). Finally, structure 2_7, being the least dense of this family $\left(F_{D}=8.60\right)$, has $\left[3^{4} 6^{4}\right]$ units ('truncated tetrahedra') linked via chains of four-rings and $\left[3^{4}\right]$ units (Fig. 9d). This very open cubic structure has $16-\mathrm{MR}$ pores in all three dimensions.

\subsection{Orphan structures}

We show three selected structures out of the 12 which cannot be categorized in our 'family' system.

Structure 2_96, a feasible zeolite structure $(\vartheta=5.45)$, is unusual as it contains small $\left[4^{5} 5^{2} 6^{2}\right]$ cage units (Fig. 9e) interconnected through shared four-rings to form a threedimensional network (Fig. 9f), thereby defining the $\left[5^{12} 6^{20}\right]$ cage which also appears in structure 2_97.

Structure 2_37 (Figs. 9h-j): the basic building unit is the D4R, which links via four-rings to create double 12-membered rings (Fig. $9 i$ ), which are in turn linked into 
large $\left[4^{36} 8^{4} 12^{8}\right]$ supercages with tetrahedral symmetry, with four puckered 12-rings and four 12-rings which are almost planar (Fig. 9j). This cubic structure is quite open with $F_{D}=$ $12.58 T$ per $1000 \AA^{3}$, but is of intermediate feasibility $(\vartheta=$ 18.50).

Structure 2_94 (Figs. $9 k$ and $l$ ) contains $\left[3^{4} 6^{4}\right]$ truncated tetrahedra, distorted sodalite cages, and larger cages with three- and six-rings (Fig. 9l). The ideal symmetry of the structure is $F d \overline{3} m$. However, in silica form it appears highly strained in this symmetry, preferring to minimize in space group $C 2$, giving rise to its somewhat distorted appearance.

\section{Conclusions}

We have evaluated and characterized 109 hypothetical zeolite structures, of which 98 do not correspond to known zeotype frameworks. Among these are many promising candidates for (a)

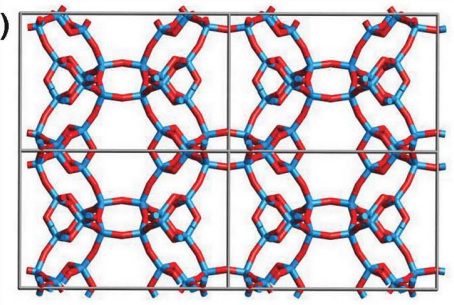

2_16

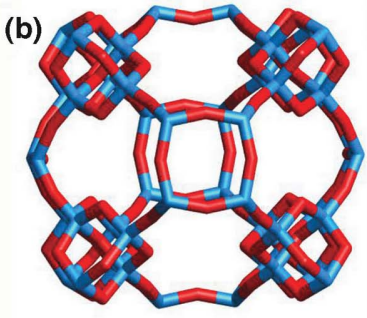

2_16

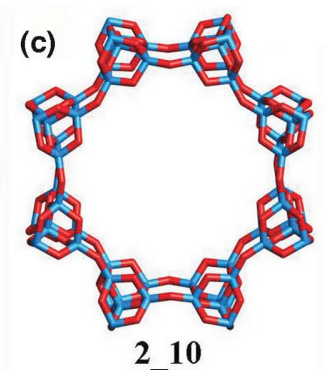

2_10

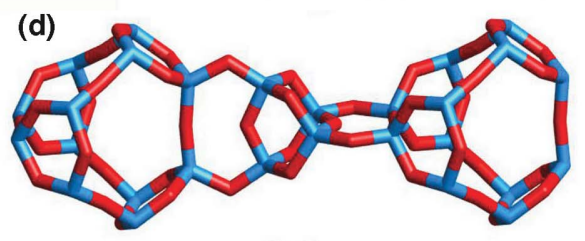

2_7 (e)

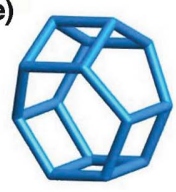

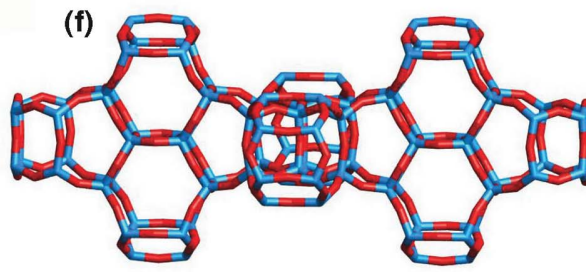

2_96

(h)

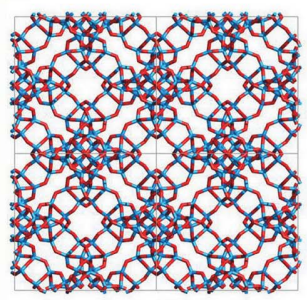

(k)

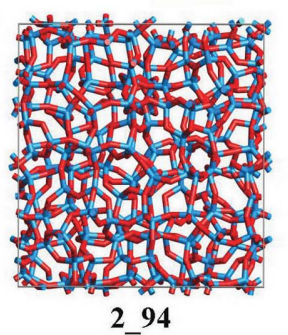

(g)

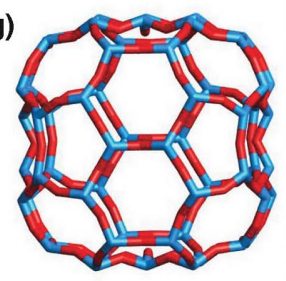

(j)

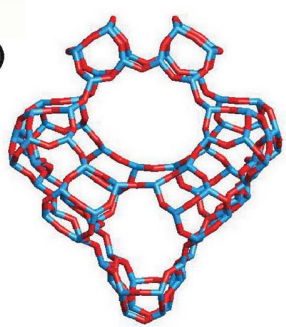

(I)

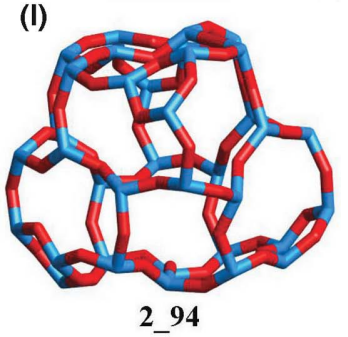

Figure 9

Molecular graphic illustrations of structures from the $\left[3^{4}\right]$ family and of some of the orphan structures. zeolite synthesis. Some of the most feasible as conventional aluminosilicates or AIPOs are those in the ABC-6 family, composed principally of four- and six-rings, although from the point of view of porosity, the more likely structures will be at best small-pore zeolites, having no aperture larger than the eight-ring. Other promising candidates come from structures which similarly have features in common with known zeolites, such as those in the AWW and SAS families (Figs. 5 and 6), where cages stack through shared eight-rings. Again, four- and six-rings predominate, with the eight-ring being the limiting aperture in all cases, as it is for the more feasible structures in the $\left[4^{2} 5^{6}\right]$ family. At the other end of the scale, many very open structures also exist. These illustrate well the principle (Brunner \& Meier, 1989) that less dense structures require a greater proportion of small (three- or four-membered) rings. Here, we can extend this to state that larger cavities also require the presence of much smaller cages. Hence, we find largepore structures containing $\left[3^{4}\right]$ units (Fig. 9), double three-rings (Fig. 8) and three-rings, as well as pairs and chains of three- and fourrings. In terms of aluminosilicate and aluminophosphate zeolites, these structural units, particularly those containing three-rings, are by and large disfavoured due to the strain imposed on the $\mathrm{TO}_{4}$ tetrahedra. In fact, it is apparent that feasibility decreases markedly as more three-rings are connected together with, for example, structures containing $\left[3^{4}\right]$ units having higher values than those containing only spiro-5 units. The most viable 
three-ring structures are those in which the three-rings are isolated from one another. The best example is 2_103 which contains the $\left[3^{2} 5^{6}\right]$ unit (Fig. $4 g$ ), reminiscent of the $\left[3^{1} 4^{3} 5^{3}\right]$ units in the MEI structure. Structure 2_103 is the most feasible large-pore zeolite among our 109 structures. Similarly, although four-rings are found in the most feasible structures, agglomerations of these units, obtained by stacking prismatic units such as D4R and D6R, result in decreasing likelihood (although individual $\mathrm{D} 4 \mathrm{R}$ and $\mathrm{D} 6 \mathrm{R}$ are tolerated, unlike D3R).

Having discounted many of the more open structures as potential zeolites on account of the presence of these small units, we do not exclude the possibility that these topologies could be possible in other chemical compositions where the local coordination environments are less constrained. Indeed, if we could construct units such as the D3R or the supertetrahedron as precursor species, many open framework architectures could be synthesized.

We are grateful to the EPSRC (U.K.) and to the Leverhulme Trust for support, and to the Portuguese Foundation for Science and Technology (FCT) for the Ph.D. scholarship No. SFRH/BD/3024/2000 to F.A.A.P.

\section{References}

Akporiaye, D. E. \& Price, G. D. (1989). Zeolites, 9, 23-32.

Alberti, A. (1979). Am. Mineral. 64, 1188-1198.

Baerlocher, C. \& McCusker, L. B. (2004). http://www.iza-structure.org/databases.

Baerlocher, C., Meier, W. M. \& Olson, D. H. (2001). Atlas of Zeolite Structure Types (updates at http://www.iza-structure.org/), 5th ed. London: Elsevier.

Barrer, R. M. \& Villiger, H. (1969). Z. Kristallogr. 128, 352-370.

Bialek, R. (1995). KRIBER, Version 1.1. Institut für Kristallographie und Petrographie, ETH, Zürich, Switzerland.

Boisen, M. B., Gibbs, G. V., O'Keeffe, M. \& Bartelmehs, K. L. (1999). Micropor. Mesopor. Mater. 29, 219-266.

Brunner, G. O. \& Meier, W. M. (1989). Nature, 337, 146-147.

Connolly, M. L. (1985). J. Am. Chem. Soc. 107, 1118-1124.

Delgado Friedrichs, O. (2001). Discret. Comput. Geom. 26, 549-571.

Delgado Friedrichs, O., Dress, A. W. M., Huson, D. H., Klinowski, J. \& Mackay, A. L. (1999). Nature, 400, 644-647.

Dress, A. W. M., Huson, D. H. \& Molnár, E. (1993). Acta Cryst. A49, 806-817.

Foster, M. D., Bell, R. G. \& Klinowski, J. (2001). Stud. Surf. Sci. Catal. 136, 266.

Foster, M. D., Delgado Friedrichs, O., Bell, R. G., Almeida Paz, F. A. \& Klinowski, J. (2003). Angew. Chem. Int. Ed. 42, 3896-3899.

Foster, M. D., Friedrichs, O. D., Bell, R. G., Almeida Paz, F. A. \& Klinowski, J. J. (2004). Am. Chem. Soc. 126, 9769-9775.

Foster, M. D., Simperler, A., Bell, R. G., Delgado Friedrichs, O., Almeida Paz, F. A. \& Klinowski, J. (2004). Nature Mater. 3, 234238.

Foster, M. D. \& Treacy, M. M. J. (2004). Hypothetical Zeolites: Enumeration Research; http://www.hypotheticalzeolites.net/ 2004.

Henson, N. J., Cheetham, A. K. \& Gale, J. D. (1994). Chem. Mater. 6, $1647-1650$.
Hu, Y. T., Navrotsky, A., Chen, C. Y. \& Davis, M. E. (1995). Chem. Mater. 7, 1816-1823.

Klinowski, J. (1998). Curr. Opin. Solid State Mater. Sci. 3, 7985 .

Koningsveld, H. van (2004). Schemes for Building Zeolite Structure Models, in Database of Zeolite Structures; http://topaz.ethz.ch/IZASC/ModelBuilding.htm.

Liebau, F., Gies, H., Gunawardane, R. P. \& Marler, B. (1986). Zeolites, 6, 373-377.

Meier, W. M. (1986). Zeolites and Zeolite-Like Materials. 7th Int. Zeolite Conference, Tokyo, 17-22 August.

Meier, W. M. \& Villiger, H. (1969). Z. Kristallogr. 128, 352370.

Mellot-Draznieks, C., Girard, S., Férey, G., Schön, J. C., Cancarevic, Z. \& Jansen, M. (2002). Chem. Eur. J. 8, 4103-4113.

Mellot-Draznieks, C., Newsam, J. M., Gorman, A. M., Freeman, C. M. \& Férey, G. (2000). Angew. Chem. Int. Ed. 39, 2270-2275.

Molecular Simulations Inc. (1999). Cerius ${ }^{2}$, Version 4.0. Molecular Simulations Inc., San Diego, USA.

Moloy, E. C., Davila, L. P., Shackelford, J. F. \& Navrotsky, A. (2002). Micropor. Mesopor. Mater. 54, 1-13.

O'Keeffe, M. \& Hyde, S. T. (1996a). Z. Kristallogr. 211, 7378.

O'Keeffe, M. \& Hyde, B. G. (1996b). Crystal Structures I: Patterns and Symmetry. Mineralogical Association of America Monograph, Washington, DC.

Navrotsky, A., Petrovic, I., Hu, Y. T., Chen, C.-Y. \& Davis, M. E. (1995). Micropor. Mater. 4, 95-98.

Persistence of Vision Raytracer Pty. Ltd (2004). POV-Ray, Version 3.6. Persistence of Vision Raytracer Pty Ltd.

Petrovic, I., Navrotsky, A., Davis, M. E., Zones, S. I. (1993). Chem. Mater. 5, 1805-1813.

Piccione, P. M., Laberty, C., Yang, S. Y., Camblor, M. A., Navrotsky, A. \& Davis, M. E. (2000). J. Phys. Chem. B, 104, 1000110011.

Piccione, P. M., Woodfield, B. F., Boerio-Goates, J., Navrotsky, A. \& Davis, M. E. (2001). J. Phys. Chem. B, 105, 6025-6030.

Piccione, P. M., Yang, S. Y., Navrotsky, A. \& Davis, M. E. (2002). J. Phys. Chem. B, 106, 3629-3638.

Sastre, G. \& Gale, J. D. (2001). Micropor. Mesopor. Mater. 43, 27 40.

Sato, M. (1984). Framework Topology and Systematic Derivation of Zeolite Structures, edited by D. H. Olson and A. Bisio. In Proc. of the 6th Intl Zeolite Conference, Reno, USA, 10-15 July. Guildford: Butterworths.

Sato, M. J. (1987). Phys. Chem. 91, 4675-4681.

Sherman, J. D. \& Bennett, J. M. (1973). Molecular Sieves, edited by W. M. Meier and J. B. Uytterhoeven, Vol. 121, p. 52. Washington, DC: American Chemical Society.

Simperler, A., Foster, M. D., Bell, R. G. \& Klinowski, J. (2004). J. Phys. Chem. B, 108, 869-879.

Smith, J. V. (1988). Chem. Rev. 88, 149-182.

Smith, J. V. (1993). ACS Abstr. 205, 157-IEC.

SourceForge (2004). GDIS, Version 0.84. SourceForge.

Treacy, M. M. J., Randall, K. H., Rao, S., Perry, J. A. \& Chadi, D. J. (1997). Z. Kristallogr. 212, 768.

Wells, A. F. (1977). Three-Dimensional Nets and Polyhedra. New York: Wiley.

Wells, A. F. (1979). Further Studies of Three-Dimensional Nets, American Crystallographic Association Monograph No. 8, Vol. 9. Pittsburgh, PA: Polycrystal Book Service.

Wells, A. F. (1984). Structural Inorganic Chemistry, 5th ed. Oxford University Press. 